03,12

\title{
Моделирование структурных и энергетических характеристик атомов в 2D-кристалле GaS c точечными дефектами
}

\author{
(ㄱ М.М. Асадов ${ }^{1}$, С.Н. Мустафраева ${ }^{2}$, С.С. Гусейнова ${ }^{2}$, В.Ф. Лукичев ${ }^{3}$, Д.Б. Тагиев ${ }^{1}$ \\ ${ }^{1}$ Институт катализа и неорганической химии им. М.Ф. Нагиева НАНА, \\ Баку, Азербайджан \\ ${ }^{2}$ Институт фризики НАНА, \\ Баку, Азербайджан \\ ${ }^{3}$ Физико-технологический институт им. К.А. Валиева РАН, \\ Москва, Россия \\ E-mail: mirasadov@gmail.com
}

Поступила в Редакцию 31 июля 2021 г.

В окончательной редакции 5 сентября 2021 г.

Принята к публикации 6 сентября 2021 г.

\begin{abstract}
В рамках теории функционала плотности (DFT) моделированы свойства гексагонального моносульфида галлия $(\mathrm{GaS})$ с учетом влияния вакансий, связанных с ближнеупорядоченной структурой. Показано, что электронное облучение монослоя $\mathrm{GaS}$ приводит к уменьшению проводимости за счет образования точечных дефектов. Рассчитаны зонная структура, плотность состояний и энергетические свойства суперъячеек $\mathrm{GaS} \mathrm{c}$ 36 и 48 атомами с моновакансиями. DFT-расчетами получены значения энергии образования GaS и вакансий атомов $\mathrm{Ga}$ и $\mathrm{S}$, а также определена степень влияния вакансий на свойства. С учетом фазовой диаграммы $\mathrm{Ga}-\mathrm{S}$ изучено влияние состава соединения $\mathrm{GaS}$ на величину химического потенциала.
\end{abstract}

Ключевые слова: моделирование, DFT-расчет, суперъячейки GaS, точечные дефекты, энергетическая структура, плотность состояний, энергия образования, химический потенциал.

DOI: $10.21883 /$ FTT.2022.01.51830.182

\section{1. Введение}

Интерес к двумерным (2D) слоистым материалам на основе полупроводниковых соединений типа $\mathrm{A}^{\mathrm{III}} \mathrm{B}^{\mathrm{VI}}$, для которых характерны квантовые эффекты, обусловлен потенциалом их применения в наноразмерных устройствах $[1,2]$. В 2D-кристаллах $\mathrm{A}^{\mathrm{III}} \mathrm{B}^{\mathrm{VI}}$ в слоях действует ковалентная связь за счет перекрывания валентных орбиталей, а между слоями - слабая ван-дер-Ваальсова связь (несколько $\mathrm{kJ} / \mathrm{mol}$ ) [3]. Такая связь между слоями может формироваться, в частности, за счет дисперсионного эффекта (взаимодействие мгновенных диполей, возникающих в атомах (или молекулах) из-за временного смещения электронных орбит и ядер). Слоистый полупроводниковый моносульфид галлия $(\mathrm{GaS})$ представляет собой материал с непрямой запрещенной зоной $\Delta E_{g}$ ), равной $\sim 2.53 \mathrm{eV}$ при $77 \mathrm{~K}[3]$. GaS имеет возможные применения в оптоэлектронных устройствах в видимом диапазоне и изучается довольно длительное время. Результаты физико-химических [3] и физических [4] исследований $\mathrm{GaS}$ систематизированы в справочниках. Информация о влиянии примесей на свойства $\mathrm{GaS}$ для изготовления высококачественных устройств, в частности, приведена в работе [5]. Соединение $\mathrm{GaS}$ в одной из модификаций имеет гексагональную слоистую структуру типа $\mathrm{GaS}[3,4,6,7]$.

$\mathrm{GaS}$ имеет ряд преимуществ перед другими 2D-материалами: широкая область рабочих температур, возможность создавать на их основе светоизлучающие устройства в широком спектре, высокие значения критического поля электрического пробоя, радиационная стойкость. В слоистом кристалле $\mathrm{GaS}$ имеются связи как $\mathrm{Ga}-\mathrm{S}$, так и Ga-Ga. Каждый слой состоит из пакетов S-Ga-Ga-S, где каждый атом галлия тетраэдрически окружен тремя атомами серы и одним атомом галлия. Пакеты S-Ga-Ga-S могут иметь различную укладку, что объясняет существование политипов моносульфида галлия. $\mathrm{GaS}$ имеет высокие фотоэлектрические, оптоэлектронные и анизотропные свойства. В зависимости от наложения слоев в кристаллической структуре $\mathrm{GaS}$ различают четыре политипа $\mathrm{GaS}(\beta, \varepsilon, \gamma$ и $\delta)$. Гексагональная фаза $\mathrm{GaS}$ (пространственная группа (пр.гр.) $P 6_{3} / m m c$ ) [1,7], т.е. $\beta$-политип $\mathrm{GaS}$ является энергетически выгодным кристаллическим расположением. При комнатной температуре термодинамически стабильной модификацией является $\beta$-GaS. Один слой $\beta$-GaS состоит из повторяющихся единиц S-Ga-Ga-S, образованных шестичленными кольцами $\mathrm{Ga}_{3} \mathrm{~S}_{3}$. При этом смежные слои $\mathrm{GaS}$ удерживаются вместе вдоль $c$-оси кристалла силами Ван-дер-Ваальса.

Распределение плотности электронов в гексагональной элементарной ячейке $\mathrm{GaS}$ (пространственная группа (пр. гр. $\left.\left.P 6_{3} / m m c\right), a=3.592(1) \AA, c=15.465(3) \AA\right)$ изучено экспериментально и методом псевдопотенциала с учетом связи между ближайшими соседями Ga-Ga $(2.449 \AA)$ и $\mathrm{Ga}-\mathrm{S}$ в кристалле [7]. Показано, что в $\mathrm{GaS}$ по экспериментальным данным существуют химические связи не только между ближайшими соседними атомами $\mathrm{Ga}-\mathrm{S}$ и $\mathrm{Ga}-\mathrm{Ga}$ в элементарной ячейке, но также и связи между $\mathrm{Ga}$ и $\mathrm{S}$ со следующими ближайшими соседями 
в той же элементарной ячейке и с атомами в окружающих элементарных ячейках. Метод псевдопотенциала не может предсказать тип указанных химических связей в $\mathrm{GaS}[8,9]$. Это связывается с необычной электронной плотностью в $\mathrm{GaS}$, где атомы не плотно упакованы и валентности (группы III и VI) компонентов не совпадают.

Изучение свойств, структуры и возможности применения наноразмерных 2D-полупроводников типа GaS методами теории функционала плотности (DFT) продолжаются. В приближение локальной плотности (LDA) в работе [8] рассчитан фононный спектр кристалла $\beta$-GaS. Определены упругие постоянные и скорости звука вдоль и поперек слоев. А в работе [9] неэмпирически рассчитаны зависимости энергии сворачивания и ширины запрещенной зоны нанотрубок $\mathrm{GaS}$ от их диаметра. Показано, что зависимость энергии сворачивания от диаметра удовлетворяет закону обратных квадратов и одинакова для нанотрубок типов „зигзаг“ и „кресло“.

В наноматериалах на основе $\mathrm{GaS}$ также проявляются анизотропные свойства. Соединения со структурой типа $\mathrm{GaS}$ по оптическим и электрическим свойствам [10-13] близок к 2D-материалам нанотехнологии, например, таким, как графен [14] и топологические изоляторы [15].

В отличие от других монохалькогенидов металлов, например, GaSe $[9,10]$, при комнатной температуре объемные кристаллы $\mathrm{GaS}$ имеют более широкую оптическую запрещенную зону $\left(\Delta E_{g}\right)$ (при $300 \mathrm{~K}$ : непрямая $\Delta E_{g} \sim 2.5-2.6 \mathrm{eV}[16]$, а прямая $\left.\Delta E_{g} \sim 3.0 \mathrm{eV}[17]\right)$. Величина $\Delta E_{g}$ резко возрастает выше $3 \mathrm{eV}$ с уменьшением толщины $\mathrm{GaS}$ до монослоя из-за эффектов квантового ограничения [18]. Таким образом, $\mathrm{GaS}$ по свойствам размещается между 2D-полупроводниками с малой и изоляторами. Это представляет интерес для реализации ультрафиолетовых (УФ) селективных фотоприемников [19], настраиваемых по цвету синий/УФ-светодиод (LEDsсветодиоды) и гетеропереходов в фотокатализе [20-22].

Создание способов контроля дефектной структурой материалов является важной задачей. Изучение влияния дефектов структуры на физико-химические свойства 2D-материалов важно для микро- и наноэлектроники. Исследования разупорядочения точечных дефектов, размеры которых находятся в нанометровом диапазоне, в кристаллических материалах обычно проводятся экспериментальными методами, например, изучаются зависимости свойств от состава и структуры материала. Однако в полупроводниковых соединениях вышеуказанная задача осложняется наличием ориентации дефектов, неэквивалентных катионных и анионных подрешеток [23]. Ограничения в применении экспериментальных способов приводят к неоднозначной интерпретации природы и местоположения дефектов.

Наряду с экспериментальными исследованиями дефектов развиваются и теоретические подходы. Атомистические модели, которые основываются на полуэмпирической параметризации межатомных сил (межатомные потенциалы), термодинамические и кристаллохимические подходы не всегда позволяют удачно решать задачи, связанные с дефектами структуры [24]. Межатомные потенциалы обычно создаются с использованием ограниченного набора параметров (энергия когезии, упругие постоянные и энергии дефектов упаковки), характеризующих материал. А термодинамические методы предполагают наличие базы данных по энергиям образования и стабилизации катионов и анионов в определенных кристаллографических позициях. Применение таких подходов часто оказывалось неверным с точки зрения предсказанных энергий образования и миграции, а также атомных конфигураций дефектов.

Расчеты на основе теории функционала плотности значительно улучшили качество прогнозов свойств в дефектных кристаллах. При DFT-расчетах обычно опираются на количественную основу моделирования атомного разупорядочения в твердом теле и учитывают влияния внешних воздействий на структуру [25]. DFT-расчеты дефектных структур выполняются с использованием известных компьютерных программ. Моделирование свойств позволяет оценить природу, концентрацию дефектов, кристаллографическое разупорядочение атомов и дефектов. Согласно результатам $a b$ initio расчетов, например, в работе [26] показано, что с уменьшением количества слоев $\mathrm{GaS}$ ширина запрещенной зоны $\mathrm{GaS}$ увеличивается $\left(\Delta E_{g} \approx 3.325 \mathrm{eV}\right)$.

Поведение дефектов в 2D-материалах изучено недостаточно, тогда как влияние дефектов на поверхностные состояния могут быть существенными. Состояние поверхности материала также может существенно влиять на поведение дефектов. В полупроводниковых соединениях возможно существование разных типов дефектов, в частности, таких как нейтральные и заряженные атомные вакансии, межузельные атомы, дефекты типа Шоттки и Френкеля.

Многие соединения типа $\mathrm{A}^{\mathrm{III}} \mathrm{B}^{\mathrm{VI}}$ имеют нестехиометрический состав [3]. Поэтому, например в $\mathrm{GaS}$, дефициты серы и галлия, проявляющиеся в виде точечных дефектов (вакансий) серы и галлия, могут влиять на энергетическую атомную конфигурацию и проводимость $\left(\sigma_{\|}=4 \cdot 10^{-13} \Omega^{-1} \cdot \mathrm{cm}^{-1} ; \sigma_{\perp}=8 \cdot 10^{-8} \Omega^{-1} \cdot \mathrm{cm}^{-1}\right.$ при $400 \mathrm{~K}$ [4]). Как известно, дефекты влияют на свойства материалов. Поэтому изучение взаимодействия дефектов в атомном масштабе, важно для описания электронной и энергетической структуры. Реакции между дефектами в атомной решетке можно рассмотреть аналогично химическим реакциям между молекулами. Это сходство связано с тем, что дефекты можно рассматривать как квазичастицы, которые характеризуются своим положением, скоростью, массой и эффективной подвижностью [23].

С учетом вышеуказанного, актуальным является выбор методов DFT и современных моделей межатомных сил при моделировании межмолекулярного взаимодействия с участием дефектов [25]. Важными являются следующие два аспекта изучения дефектообразования в кристаллах. Во-первых, дефекты, образовавшиеся в результате нарушения совершенства кристаллических решеток структуры, малы и, следовательно, не доступ- 
ны для прямого экспериментального наблюдения. Вовторых, упрощенные модели межатомных сил, использовавшиеся для моделирования дефектов до того, как стали доступны методы DFT, недостаточно точны и надежны. Например, структура дефектов в объемноцентрированных кубических металлах, предсказанная с помощью эмпирических потенциалов, оказалась неверной. Также оказалось неверным описание природы взаимодействия между вакансиями; указанное отличалось от того, что было позже обнаружено с использованием методов DFT.

Кроме указанного, до сих пор нет $a b$ initio информации об энергии образования и миграции вакансий в соединениях типа $\mathrm{A}^{\mathrm{III}} \mathrm{B}^{\mathrm{VI}}$. Цель настоящей работы DFT-расчет структурных и энергетических характеристик атомов в суперъячейках $\mathrm{GaS}$ с упорядоченными точечными дефектами.

\section{2. Методика эксперимента и расчета}

Синтез GaS. Поликристаллы $\mathrm{GaS}$ синтезированы с использованием метода химического взаимодействия высокочистых исходных элементов (Ga и $\mathrm{S}$ ) в вакуумированной кварцевой ампуле. По данным рентгенофазового (РФА) и микроскопического анализов поликристаллы $\mathrm{GaS}$ состояли из гексагональной фазы. На картинах дифракции рентгеновских лучей обнаруживаются пики (002), (004) (006), (008) и (208) гексагональной $\beta$-фазы GaS. Дифракционные пики GaS соответствовали параметрам $a=3.583 \pm 0.002 \AA, c=15.475 \pm 0.005 \AA$ (рис. 1) и данным карты JCPDS № 30-0576 для $\beta$-GaS (пр.гр. $P 6_{3} / m m c,(194), a=3.587 \AA$ и $c=15.492 \AA$ ).

Монокристалл нужного политипа $\mathrm{GaS}$ со стехиометрическим составом трудно выращивать из-за летучести халькогена при температурах выращивания (<1230 K). Монокристаллы $\mathrm{GaS}$ получали методом Бриджмена [3]. В качестве эталона структуры для сравнения GaS использовали известные данные GaS (карта JCPDS № 300576). Выращенные нами монокристаллы $\mathrm{GaS}$ показали

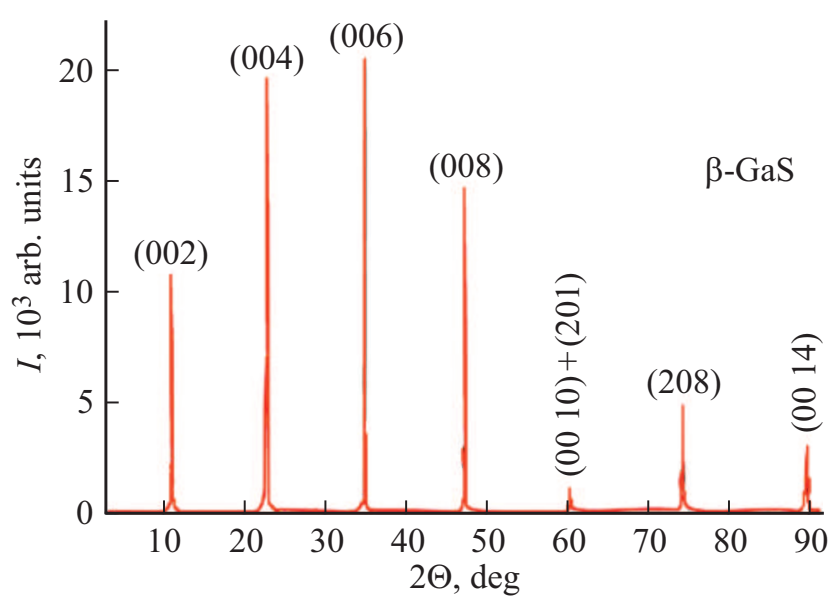

Рис. 1. Дифрактограмма порошкового образца монокристалла $\mathrm{GaS}$ при $298 \mathrm{~K}$. высокие фотоэлектрические и диэлектрические свойства. Монокристаллы GaS были желтого цвета. После отжига при $1273 \mathrm{~K}$ в течение 5 h в вакууме эти образцы $\mathrm{GaS}$ становятся более светлого цвета. Монокристаллы $\mathrm{GaS}$, выращенные с относительно высокой скоростью роста $(>2 \mathrm{~mm} / \mathrm{h})$, содержали точечные дефекты. Структурные дефекты влияют на диэлектрические и электрические свойства $\mathrm{GaS}[1,5]$.

Вычислительная методика. Расчеты выполнены на основе метода DFT с использованием плоских волн псевдопотенциала [27-30]. Использовали суперъячейки $\mathrm{GaS}$, содержащие соответственно 36 и 48 атомов, а также вакансии атомов $\mathrm{Ga}$ и $\mathrm{S}$. Для расчета обменнокорреляционной энергии применяли как приближение локальной плотности (LDA) [27,28], так и приближение обобщенного градиента (GGA) [29] с использованием базовых наборов в различных реализациях. Значения вычисленного параметра $y_{i j}$ принимали как среднее значение отдельных расчетов $y_{i j}$. Стандартное отклонение рассчитывали по формуле $\sigma_{i}=\sqrt{\sum_{j}\left(y_{i j}-y_{i}\right)^{2}} /(n-1)$, где $n$ - общее количество расчетов $[14,30]$.

Кристаллическую структуру оптимизировали до тех пор, пока максимальное значение силы межатомного взаимодействия не стало меньше $0.01 \mathrm{eV} / \AA \AA$, а максимальное значение тензора механических напряжений не было меньше $0.01 \mathrm{eV} / \AA^{3}$.

B DFT-расчетах с участием суперъячеек $\mathrm{GaS}$ с вакансиями $\mathrm{Ga}$ или $\mathrm{S}$ для численного интегрирования по зоне Бриллюэна использовалась сетка $2 \times 2 \times 2$ $k$-точек, генерируемая схемой Монкхорста-Пака [31]. Кинетическая энергия отсечки $E_{\text {cut }}$, которая определяет сферу плоских волн, составляла $410 \mathrm{eV}$. Максимальное значение $E_{\text {cut }}$ не превышало $2040 \mathrm{eV}[14,30]$.

Самосогласованные полные энергии получены с использованием схемы смешения плотности в сочетании с методом сопряженных градиентов. Положения атомов оптимизированы с помощью алгоритма БройденаФлетчера-Гольдфарба-Шанно (algorithm BFGS) [32]. Геометрические координаты атомов в суперъячейке оптимизировали с учетом сопряженных градиентов путем вычисления сил, действующих на атомы. Релаксацию проводили путем минимизации сил, действующих на каждый атом. Кристаллическую ячейку $\mathrm{GaS}$ оптимизировали с допуском к силе $0.01 \mathrm{eV} / \AA$ и напряжению $0.001 \mathrm{eV} / \AA^{3}$.

Расчет энергии образования вакансий. В DFTрасчете дефект в виде вакансии $(V)$ формируется путем удаления атома из узла решетки в суперъячейке и ослабления полученной атомной структуры. Энергия образования вакансии в общем виде рассчитывается по уравнению

$$
E_{f}^{V}=E_{\text {tot }}^{N-1(\text { relaxed })}-\left(\frac{N-1}{N}\right) E_{\text {tot }}^{N(\text { perfect })},
$$

где $E_{\mathrm{tot}}^{N-1 \text { (relaxed) }}$ - полная энергия суперъячейки (релаксированная) с удаленным одним атомом, $E_{\text {tot }}^{N(\text { perfect })}-$ 
полная энергия ячейки (совершенная) с атомами без дефектов. Индексы $N-1$ и $N$ относятся к общему количеству атомов в ячейке. Это уравнение показывает, что для определения энергии образования вакансии требуются два отдельных вычисления методом DFT. Чтобы найти достаточно точное значение энергии образования вакансии, требуется относительно небольшая ячейка, содержащая менее 100 атомов.

Энтальпию образования гексагональной фазы $\mathrm{GaS}$ и энергию образования нейтральных вакансий в суперъячейках $\mathrm{GaS}$ вычисляли методом DFT. Формируя нейтральные атомные вакансии в суперъячейках $\mathrm{GaS}$ определили влияние вакансий на свойства GaS. Вычисляли энергию образования нейтральных вакансий. Энтальпию образования вычисляли для модельной гексагональной структуры $\mathrm{GaS}$, формирующейся при атмосферном давлении. Расчеты энергии образования вакансий $\mathrm{Ga}$ и $\mathrm{S}$ в $\mathrm{GaS}$ проведены с использованием суперъячеек, содержащих 36 и 48 атомов. Это количество атомов элементов $\mathrm{Ga}$ и $\mathrm{S}$, соответственно в 4.5 и 5 раз больше, чем атомов в примитивной ячейке $\mathrm{GaS}$.

Расположение атомов вокруг вакансий (Ga или $\mathrm{S}$ ) суперъячеек $\mathrm{GaS}$ оптимизировали с учетом релаксации первых и вторых ближайших соседей атомов вакансии. Параметры кристаллической решетки $\mathrm{GaS}$ фиксировали на оптимизированном значении идеального кристалла.

\section{3. Результаты расчетов и обсуждение}

В целом химическая связь между разнородными атомами в твердых телах имеет ковалентно-ионнометаллический характер. В полупроводниках вклад ковалентной связи всегда является значительным. Одним из критериев того, что вещество является полупроводником, считается преобладание ковалентного вклада в химическую связь. С увеличением разности электроотрицательности возрастает вклад ионной связи. В $\mathrm{GaS}$, металлическая составляющая химической связи мала, поэтому межатомная связь в $\mathrm{GaS}$ является ковалентноионной.

Примем, что в кристалле $\mathrm{GaS}$ при дефектообразовании соблюдаются следующие ограничения. 1) Соотношение числа мест в подрешетке постоянно; 2) создание мест соответствует структурной формуле, т. е. при увеличении числа атомов в одной подрешетке в другой возникает пропорциональное структурной формуле число вакансий; 3) соблюдается баланс масс, т.е. сумма масс элементов правой и левой части квазихимических уравнений равны; 4) соблюдается электронейтральность.

Установлено, что полученный нами монокристалл $\mathrm{GaS}$ обладает высоким удельным сопротивлением $\left(\sigma_{\|} \approx 10^{-13} \Omega^{-1} \cdot \mathrm{cm}^{-1} ; \sigma_{\perp} \approx 10^{-8} \Omega^{-1} \cdot \mathrm{cm}^{-1}\right)$ и запрещенной зоной $\Delta E_{g}=2.53 \mathrm{eV}$ при $300 \mathrm{~K}$. На рис. 2 представлены частотные зависимости ас-проводимости (проводимость на переменном токе) поперек слоев в

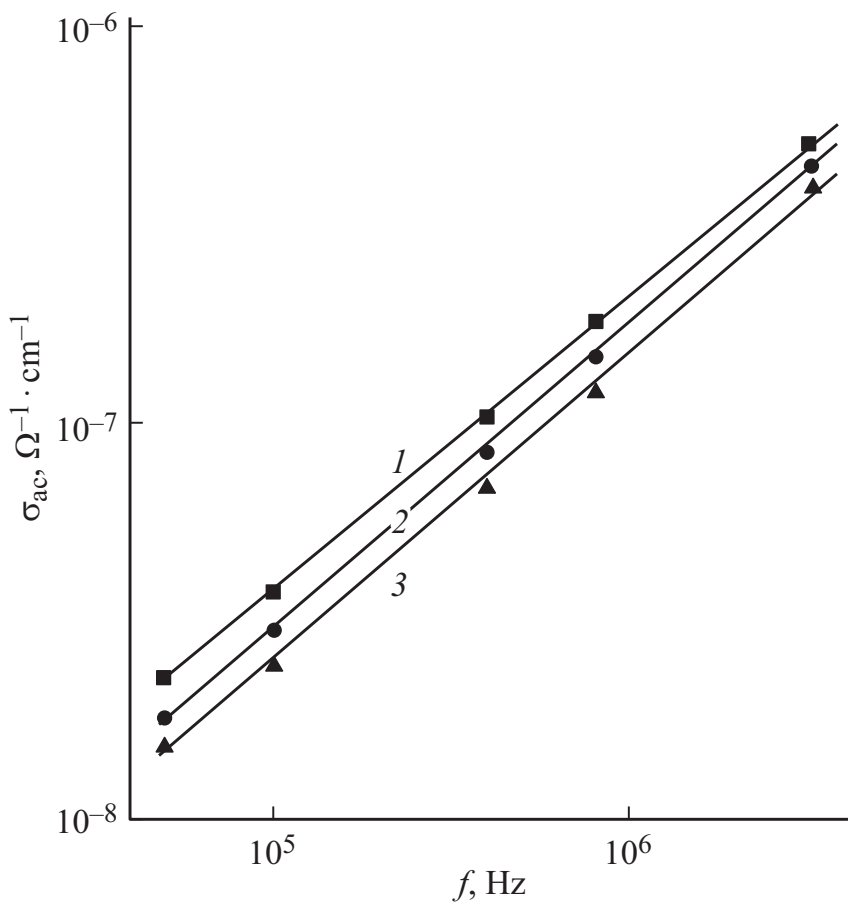

Рис. 2. Ас-проводимость монокристалла $\mathrm{GaS}$ как функция частоты: до облучения (1) и после е-облучения дозами $2 \cdot 10^{12}(2)$ и $10^{13} \mathrm{~cm}^{-2}(3)$.

выращенном монокристалле $\mathrm{GaS}$ до (кривая 1) и после электронного облучения ( $\mathrm{e}^{-}$-облучения) различными дозами (кривые 2 и 3). Как видно на рис. 2, в результате $\mathrm{e}^{-}$-облучения ас-проводимость монокристалла $\mathrm{GaS}$ уменьшается. Уменьшение электропроводности облученных полупроводников, как известно, связывают с компенсацией первоначально существовавших электрически активных примесных центров более глубокими энергетическими уровнями радиационных дефектов. В полупроводниках с глубокими энергетическими уровнями радиационных дефектов компенсация проводимости бывает резко выражена. В монокристалле $\mathrm{GaS}$, возможно, также проявляется это явление. Таким образом, облучение монокристалла $\mathrm{GaS}$ вызывает возникновение радиационных дефектов, и стимулирует отжиг и миграцию имеющихся в кристалле дефектов. Это приводит к энергетическому перераспределению локальных состояний в окрестности уровня Ферми, за счет чего по мере накопления дозы $\mathrm{e}^{-}$-облучения в образце $\mathrm{GaS}$ энергетическая полоса локализованных вблизи уровня Ферми состояний расширяется.

\section{1. Параметры решетки и зонная структура суперъячейки GaS}

Рассчитанные параметры решетки гексагональной фазы GaS получены при $0 \mathrm{~K}$, а экспериментальные значения, получены при более высоких температурах. Однако рассчитанные параметры решетки в $\mathrm{GaS}$ воспроизводили экспериментальные значения с погрешностью 2\%. Другими словами DFT расчеты для примитивной ячеки $\mathrm{GaS}$ 


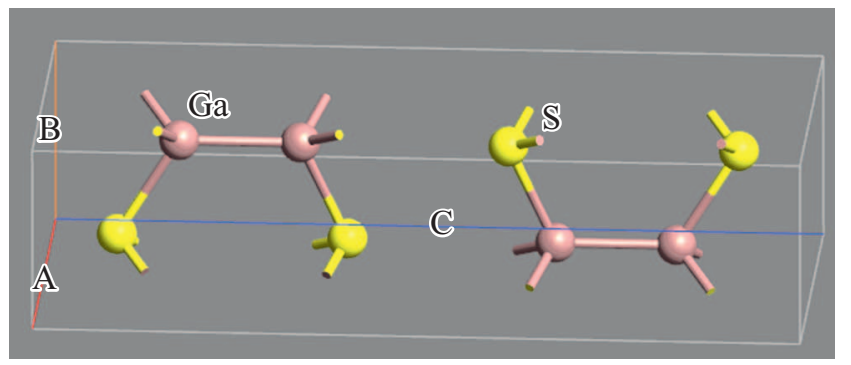

Рис. 3. Примитивная ячейка соединения $\mathrm{GaS}$.

(пр.гр. $\left.P 6_{3} / m m c,(194)\right)$ показали, что данные расчета хорошо согласуются с экспериментальными значениями (табл. 1).

Расчеты зонной структуры $\mathrm{GaS}$ для примитивной ячейки (рис. 3) в приближении локальной плотности (LDA) показали, что ширина запрещенной зоны $\left(\Delta E_{g}\right)$ является непрямой и составляет $\Delta E_{g}=1.57 \mathrm{eV}$ (рис. $4, a)$. Сравнение расчетной величины $\Delta E_{g}$ для $\mathrm{GaS}$ с экспериментальными значениями $\Delta E_{g}=2.53 \mathrm{eV}$ [18] показывает, что они сильно различаются. Рассчитанная нами методом DFT-LDA ширина запрещенной зоны GaS (рис. 4,a) оказалась меньше экспериментально полученной $\Delta E_{g}$.

Использование негибридных функционалов в DFTрасчетах обычно занижает рассчитанные значения $\Delta E_{g}$ полупроводников (так называемая „проблема поправки ширины запрещенной зоны“) [33]. В соответствие с этим можно предположить, что заполненные узкие энергетические полосы $3 d$-состояния $\mathrm{Ga}$ в $\mathrm{GaS}$ сильно связаны с $2 p$-состояниями $\mathrm{S}$, полученные из $2 p$-орбиталей серы. Тогда возможно, что $d$-состояние $\mathrm{Ga}$ может включиться в качестве валентного состояния в $\mathrm{GaS}$ и он будут влиять на структурные параметры, изменять энергетическую зону и деформационный потенциал. В противном случае, т. е. если не учесть $3 d$-состояния $\mathrm{Ga}$ в $\mathrm{GaS}$, то $3 d$-состояния будут рассматриваться как состояния с замороженным ядром.

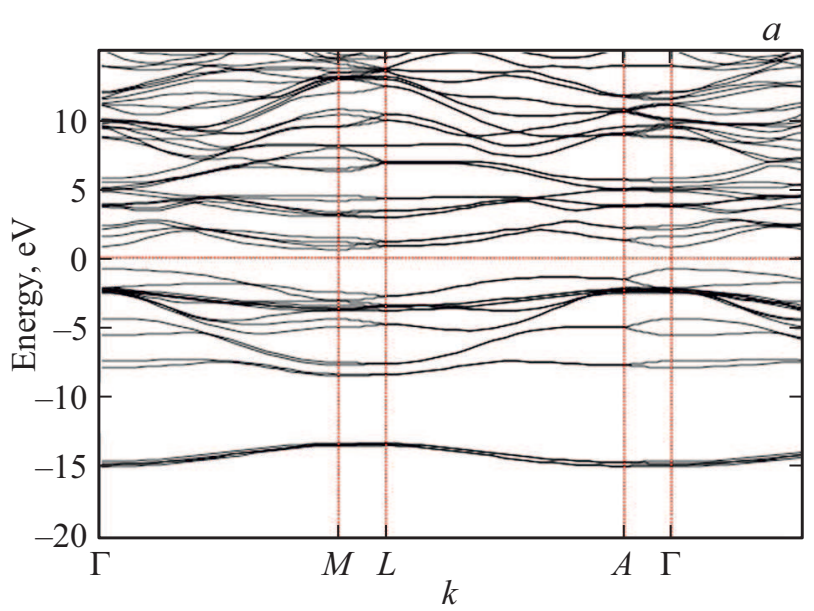

Таблица 1. Параметры решетки $\mathrm{GaS}$ в гексагональной (пр. гр. $\left.P 6_{3} / m m c\right)$ сингонии, полученные с использованием обменнокорреляционных функций

\begin{tabular}{|c|c|c|}
\hline Метод & $\begin{array}{l}\text { Параметры } \\
\text { решетки, А }\end{array}$ & $\begin{array}{c}\text { Погрешность, } \\
\%\end{array}$ \\
\hline $\begin{array}{l}\text { GGA PBE } \\
\text { (Настоящая работа) }\end{array}$ & $\begin{array}{l}a=4.002 \\
c=15.440\end{array}$ & 0.3 \\
\hline LDA (Настоящая работа) & $\begin{array}{l}a=3.957 \\
c=15.268\end{array}$ & 1.5 \\
\hline PBE [6] & $\begin{array}{l}a=3.630 \\
c=15.701\end{array}$ & 1 \\
\hline $\operatorname{PBE}[8]$ & $\begin{array}{l}a=3.617 \\
c=15.496\end{array}$ & - \\
\hline $\begin{array}{l}\text { Эксперимент } \\
\text { (Настоящая работа) }\end{array}$ & $\begin{array}{l}a=3.583 \\
c=15.475\end{array}$ & - \\
\hline Эксперимент [1,18] & $\begin{array}{l}a=3.583 \\
c=15.475\end{array}$ & - \\
\hline карта JCPDS № 30-0576 & $\begin{array}{l}a=3.587 \\
c=15.492\end{array}$ & - \\
\hline Эксперимент [3] & $\begin{array}{l}a=3.578 \\
c=15.47\end{array}$ & - \\
\hline Эксперимент [7] & $\begin{array}{l}a=3.592 \\
c=15.495\end{array}$ & - \\
\hline Справочник [4] & $\begin{array}{l}a=3.587 \\
c=15.492\end{array}$ & - \\
\hline
\end{tabular}

Расчетное значение $\Delta E_{g}$ соединения $\mathrm{GaS}$ согласовали с экспериментальными $\Delta E_{g}$. Обменную энергию рассчитывали с учетом вкладов энергии обмена и корреляции электронов в выражение функционала электронной плотности. При оценке учитывали также малое влияние $d$-состояния $\mathrm{Ga}$ на запрещенную зону GaS. Обменно-корреляционный потенциал вычислили методом GGA-PBE. Полученный результат расчета $\Delta E_{g} \approx 2.50 \mathrm{eV}$ хорошо согласуется с эксперименталь-

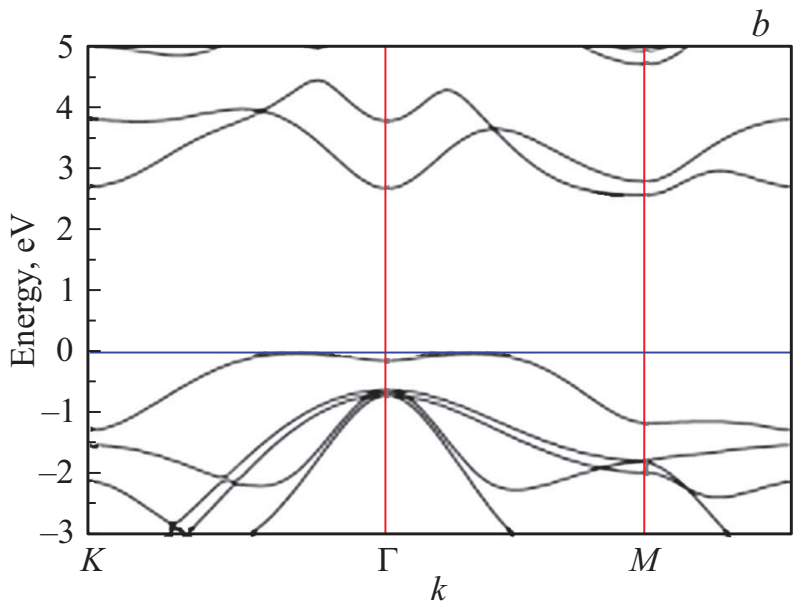

Рис. 4. Зонная структура примитивной ячейки соединения GaS. $a$ ) - приближение LDA; $b$ ) — приближение LDA + GGA. 
ным $\Delta E_{g}=2.53 \mathrm{eV}[18]$. Рассчитанные параметры решетки и $\Delta E_{g}$ для $\mathrm{GaS}$ согласуются с экспериментальными значениями в пределах погрешности $\leq 3 \%$, которая типична для DFT-расчетов. Для вычисления полной энергии в работе [9] использовали гибридный вариант метода DFT

$$
E_{\mathrm{tot}}^{\mathrm{hyb}}(1-x) E_{\mathrm{X}}^{\mathrm{HF}}+x E_{\mathrm{X}}^{\mathrm{DFT}}+E_{\mathrm{C}}^{\mathrm{DFT}},
$$

где $E_{\mathrm{tot}}^{\mathrm{hyb}}-$ полная энергия, полученная гибридным методом DFT, $1-x$ - доля энергии, полученная расчетом по методу Хартри-Фока (HF) в функционале, $E_{\mathrm{X}}^{\mathrm{HF}}-$ вклад энергии обмена по методу $\mathrm{HF}, E_{\mathrm{X}}^{\mathrm{DFT}}-$ вклад энергии обмена в негибридном (простом) варианте DFT, $E_{\mathrm{C}}^{\mathrm{DFT}}-$ вклад энергии корреляции в негибридном варианте DFT. Указано, что DFT-расчеты (содержащего 13\% обмена по Хартри-Фоку) проведены с использованием обменнокорреляционного функционала PBE. Ширина непрямой запрещенной зоны монослоя $\mathrm{GaS}$ составляет $3.30 \mathrm{eV}$, что заметно больше соответствующей величины для объемных кристаллов.

\subsection{1. Электронная структура суперьячейки GaS}

Из общего подхода описания ковалентной связи следует, что в методе молекулярных орбиталей (ММО), волновая функция $\Psi$ электронной пары характеризуется наложением волновых функций отдельных фрагментов. Взаимодействие неподеленной электронной пары атома одной молекулы $\mathrm{GaS}$ рассмотрим в рамках MMO. Примем, что волновая функция $\Psi$ для полупроводника $\mathrm{GaS}$, характеризуется орбитальной аппроксимацией и линейной комбинацией атомных орбиталей: $\Psi_{\mathrm{GaS}}=a \Psi_{\mathrm{Ga}}+b \Psi_{\mathrm{S}}$, где $\Psi_{\mathrm{Ga}}$ и $\Psi_{\mathrm{S}}-$ волновые функции, участвующие в химической связи атомов, коэффициенты $a \neq b$ - доля участия каждого атома в квантовохимической связи. Максимум электронной плотности в каждой ковалентной связи $\mathrm{GaS}$ будет смещен в сторону атома с более высокой электроотрицательностью, т.е. электронные облака в $\mathrm{GaS}$ будут стянуты к узлам кристаллической решетки, где находятся атомы серы.

Таким образом, химическую связь в 2D-кристаллах $\mathrm{GaS}$ между орбиталями $\mathrm{Ga} 4 p$ и $\mathrm{S} 3 p$ можно считать гетерополярной ковалентной. При этом происходит перераспределение электронной плотности вблизи ядер, образующих молекулу GaS. Тогда в узлах решетки будут располагаться нейтральные атомы, и связь между ними будет осуществляться посредством электронных пар. Ковалентная связь, как известно, имеет направленный характер и образуется в том направлении, где плотность электронного облака спаренных электронов наибольшая. Поэтому можно принять, что в $\mathrm{GaS}$ каждый атом кристаллической решетки может взаимодействовать только с определенными атомами, а не со всеми, как в ионных кристаллах.

С учетом вышеуказанных и теории квантовой химии валентная зона $\mathrm{GaS}$ должна будет состоять из связывающих атомных орбиталей $\mathrm{S} \quad 3 p$ и $\mathrm{Ga} 4 p$ $\left(\Psi_{+}=\Psi_{b}=\Psi_{\mathrm{Ga}}+\Psi_{\mathrm{S}}\right.$ связывающая $\left.\mathrm{MO}\right)$, где верх валентной зоны состоит из орбитали компонента $\mathrm{S} 3 p$. С другой стороны, зона проводимости $\mathrm{GaS}$ будет состоять из разрыхляющих орбиталей $\mathrm{Ga} 4 p$ и $\mathrm{S} 3 p$ $\left(\Psi_{-}=\Psi_{a}=\Psi_{\mathrm{Ga}}-\Psi_{\mathrm{S}}\right.$ разрыхляющая $\left.\mathrm{MO}\right)$, а дно зоны проводимости, включает несвязывающие орбитали, которые возникают за счет атомных орбиталей компонента Ga $4 p$.

\section{2. Вакансия $\mathrm{Ga}$ в 36-атомной суперьячейке GaS (модель $\mathrm{Ga}_{17} \mathrm{~S}_{18}$, где индексы указывают на число соответствующих атомов)}

Нестехиометрия, т.е. нарушение стехиометрического соотношения между атомами в соединениях, в частности халькогенидов металлов (MX) [3], приводит к изменению свойств и формированию дефектов в объеме МХ. Точечные дефекты в кристалле, обычно, образуются при росте, пластической деформации и термообработке материала. Степень нестехиометричности МХ, изменяют электрические свойства материала на основе МХ. Полупроводниковые халькогенидные материалы разделяют на два типа проводимости, $p$-тип и $n$-тип, которые связаны с дефицитом (или избытком) металла или с недостатком (или избытком) халькогена [3]. Примем, что в кристалле $\mathrm{GaS}$ вакансии действуют как доноры, если число неспаренных электронов вблизи вакансии меньше половины числа валентных электронов, имеющихся в аналогичном месте совершенного кристалла, и как акцепторы - в противном случае. Тогда в кристалле $\mathrm{GaS}$ между Ga и $\mathrm{S}$ создается в основном ковалентная связь с помощью восьми валентных электронов. При такой связи атомов $\mathrm{GaS}$ внешние электроны двух соседних атомов будут объединяться в пары, образуя общие орбиты вокруг обоих атомов ( $\mathrm{Ga}$ и $\mathrm{S}$ ), причем спины в каждой паре антипараллельны в силу принципа Паули. В кристалле $\mathrm{GaS}$, например, с вакансией галлия $\mathrm{Ga}\left(V_{\mathrm{Ga}}\right)$, где $V_{\mathrm{Ga}}$ будет окружена неспаренными электронами атомов серы, для связывания с ними может принимать электроны. При этом $V_{\mathrm{Ga}}$ может заряжаться отрицательно и выполнять функцию акцептора. Наоборот, вакансия серы, окруженная неспаренными электронами атомов галлия, будет донором.

Полупроводник $p$-типа с дефицитом металлов, например, такой как кристалл $\mathrm{GaS}$, можно представить как $\mathrm{Ga}_{1-x} \mathrm{~S}$ ( $x$ - атомная доля), где для любого ненулевого значения кристалл имеет набор атомных дефектов. Тогда в случае $x>0$ в кристалле будут вакансии металла $V_{\mathrm{Ga}}$, а в случае $x<0$ в кристалле будут дополнительные атомы $\mathrm{Ga}$, которые будут расположены в межузельных положениях $\mathrm{Ga}_{i}$. T.е. заряженные вакансии $F_{\mathrm{Ga}}$, будут преобладающими дефектами в богатых серой кристаллах $\mathrm{GaS}$. Кристалл $\mathrm{GaS}_{1-x}$ будет содержать вакансии ионизированной серы и междоузлия галлия.

При дефектообразовании в $\mathrm{GaS}$ могут формироваться положительно и отрицательно заряженные дефекты, 

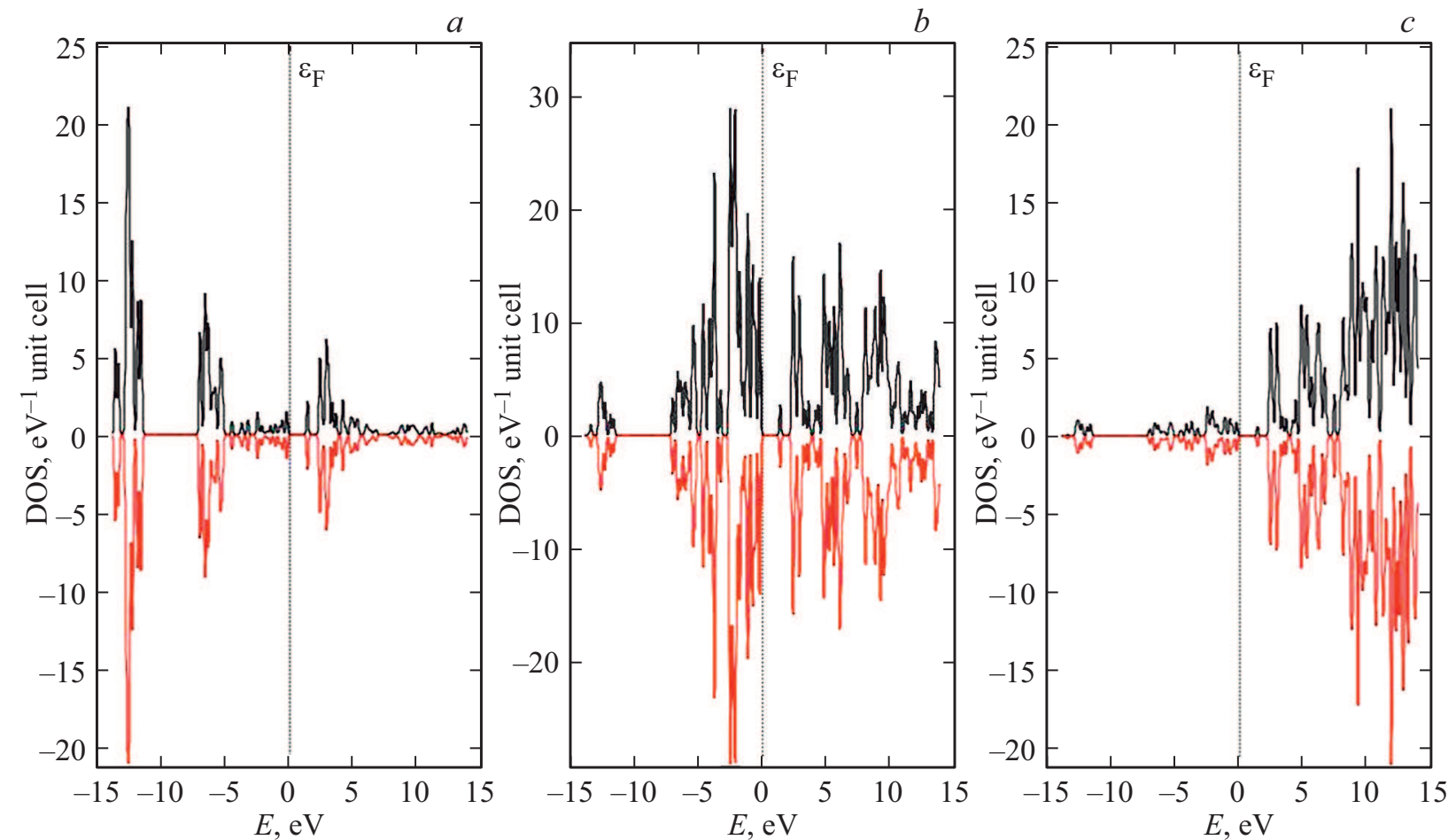

Рис. 5. Графики электронной плотности (DOS) суперъячейки GaS, содержащей Ga-вакансию (модель $\mathrm{Ga}_{17} \mathrm{~S}_{18}$ ) со спином вверх и вниз. $a) s$-состояние, $b$ ) $p$-состояние, $c) d$-состояние.
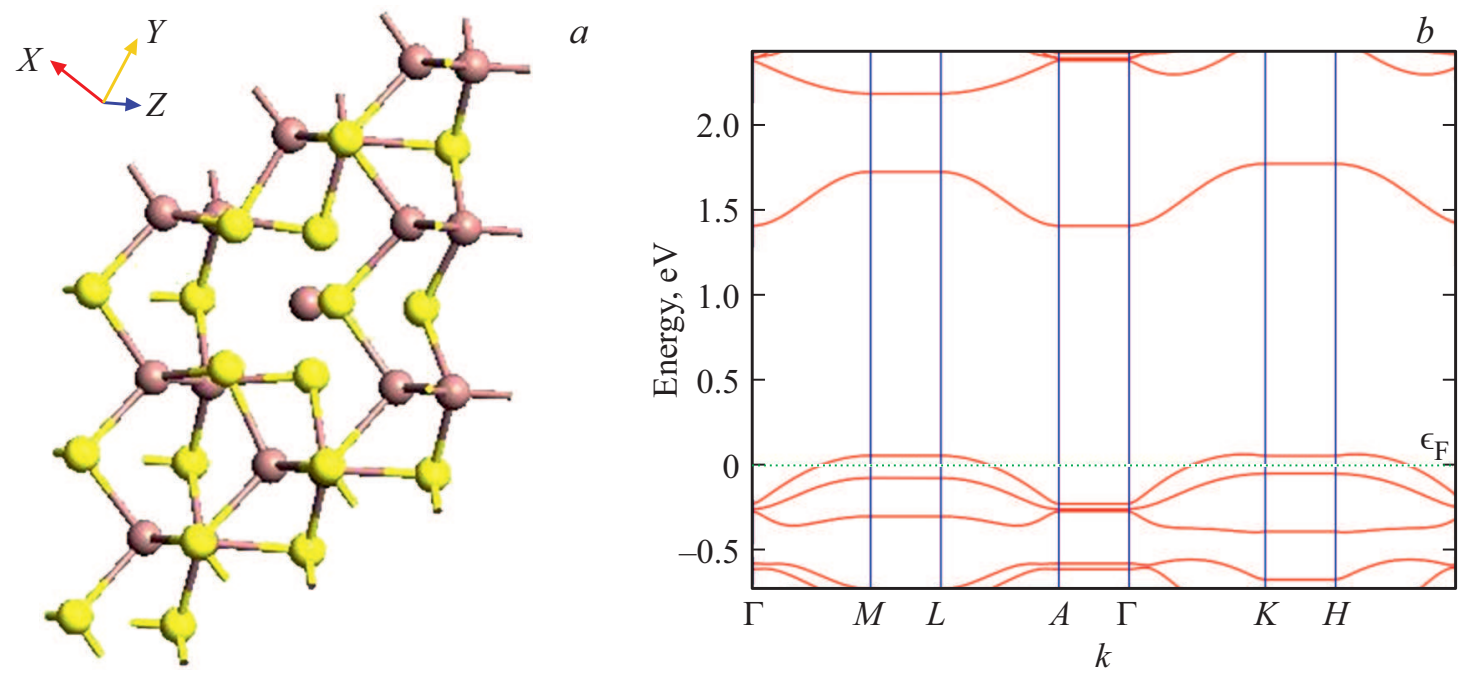

Рис. 6. Атомная $(a)$ и зонная структура $\left(b-\right.$ приближение LDA) 36-атомной суперъячейки $\mathrm{GaS}$ с вакансией $\mathrm{Ga}\left(\right.$ модель $\left.\mathrm{Ga}_{17} \mathrm{~S}_{18}\right)$.

комплексы дефектов, свободные электроны или их вакансии. Условие электронейтральности должно обеспечиваться образованием равного количества положительно и отрицательно заряженных дефектов. Рассмотрим суперъячейки $\mathrm{GaS}$ только с нейтральными точечными дефектами (вакансии $\mathrm{Ga}$ и $\mathrm{S}$ ) в соответствующих подрешетках.

На рис. 5 и 6 приведены рассчитанные плотности электронных состояний (DOS), атомная и зонная структуры суперъячейки $\mathrm{GaS}$ с вакансией галлия (модель $\mathrm{Ga}_{17} \mathrm{~S}_{18}$ ).
Результаты расчетов плотности электронных состояний в 36-атомной суперьячейке $\mathrm{GaS}$ с упорядоченной вакансией $\mathrm{Ga}$ (модель $\mathrm{Ga}_{17} \mathrm{~S}_{18}$ ) при заданных параметрах решетки (гексагональная, $a=4.002 \AA, c=15.440 \AA$, пр.гр. $\left.P 6_{c} / m m c,(194)\right)$ приведены на рис. 5.

На рис. 6 приведены атомная $(6, a)$ и зонная структуры $(6, b)$ суперьячейки $\mathrm{GaS}$ с вакансией галлия, а точнее модели суперьячейки $\mathrm{Ga}_{17} \mathrm{~S}_{18}$. Как следует из рис. $6, b$, зонная структура $\mathrm{GaS}$ с вакансией $\mathrm{Ga}$ имеет непрямую зону. 

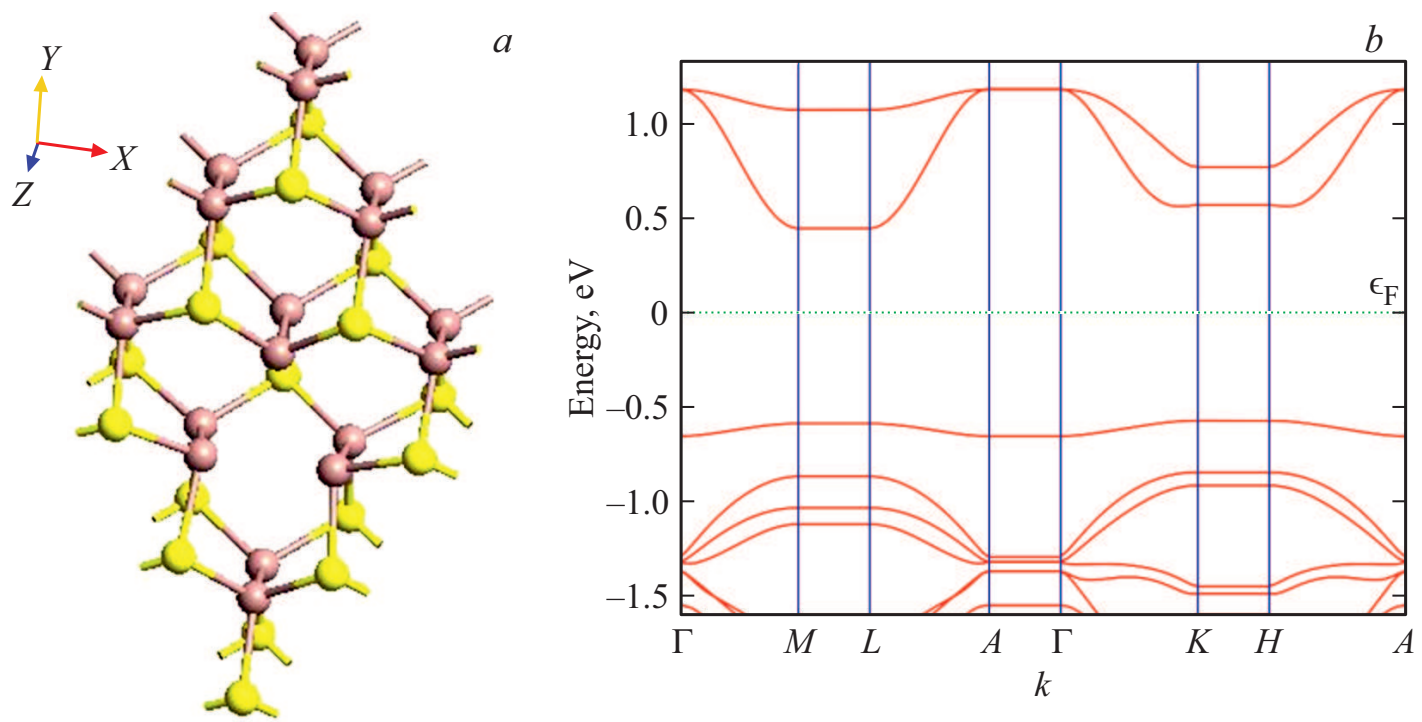

Рис. 7. Атомная $(a)$ и зонная структура ( $b$ - приближение LDA) 36-атомной суперъячейки $\mathrm{GaS}$ с вакансией $\mathrm{S}$ (модель $\left.\mathrm{Ga}_{18} \mathrm{~S}_{17}\right)$.
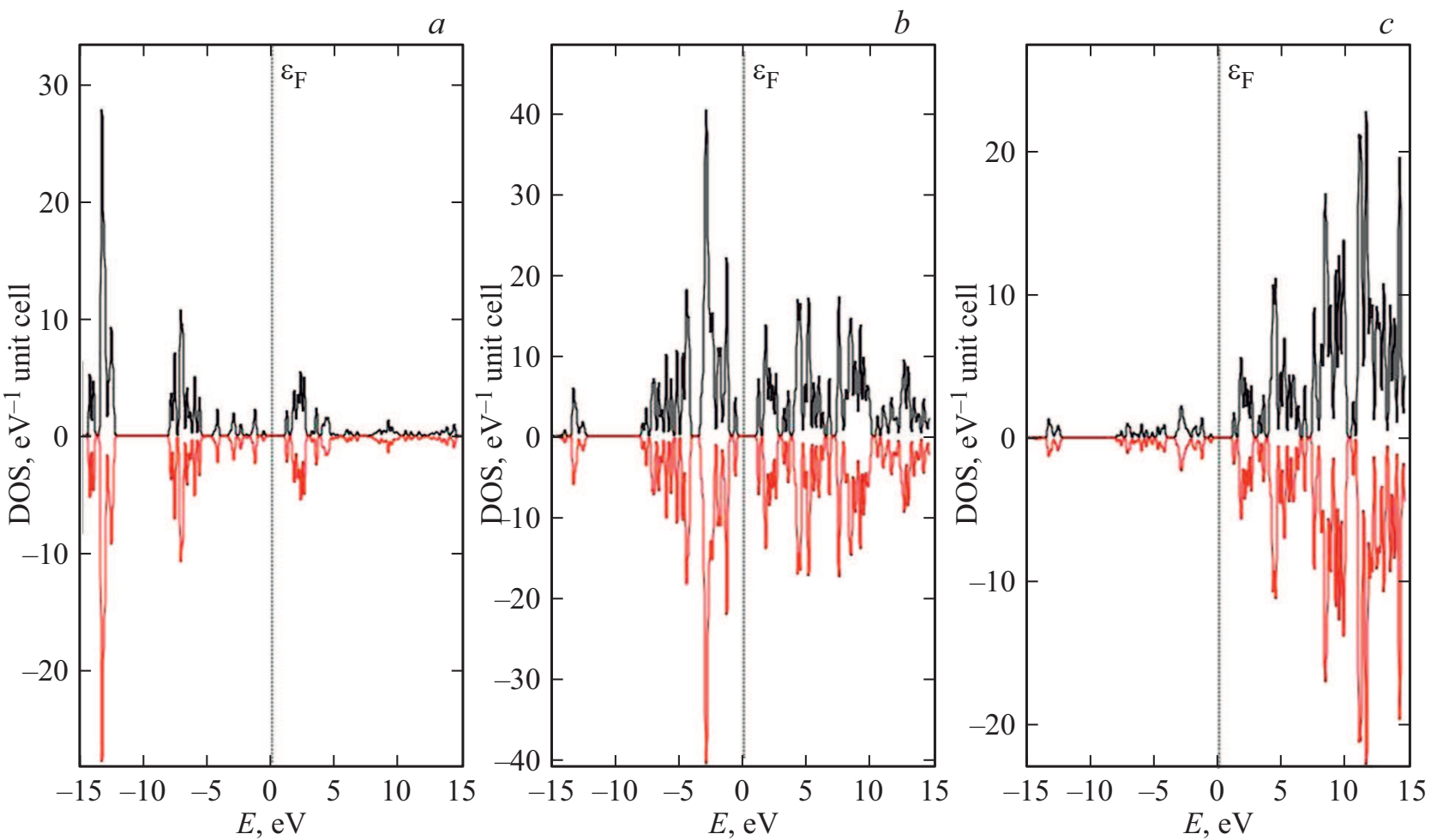

Рис. 8. Графики электронной плотности (DOS) суперьячейки GaS, содержащей S-вакансию (модель $\mathrm{Ga}_{18} \mathrm{~S}_{17}$ ) со спином вверх и вниз. a) $s$-состояние, $b$ ) $p$-состояние, $c$ ) $d$-состояние.

\section{3. Вакансия $\mathrm{S}$ в 36-атомной суперьячейке GaS (модель $\mathrm{Ga}_{18} \mathrm{~S}_{17}$ )}

На рис. 7, $a, b$ приведены атомная и зонная структуры в 36-атомной суперъячейке $\mathrm{GaS}$ с вакансией $\mathrm{S}$ (модель $\mathrm{Ga}_{18} \mathrm{~S}_{17}$ ) (гексогональная, $a=4.002 \AA, c=15.440 \AA$, пр.гр. $\left.P 6_{3} / m m c,(194)\right)$. Присутствие вакансии $\mathrm{S}$ приво- дит к уменьшению $\Delta E_{g}$. На рис. 7, $b \Gamma \rightarrow K$ зона является непрямой.

На рис. 8 показаны плотности электронных состояний 36 атомной суперъячейки $\mathrm{GaS}$ с вакансией $\mathrm{S}$. Из анализа $s$-, $p$ - и $d$-состояний DOS следует, что состояния спин вверх и спин вниз для суперъячейки $\mathrm{Ga}_{18} \mathrm{~S}_{17}$ оказываются симметричными. 


\section{4. Вакансия $\mathrm{Ga}$ в 48-атомной суперъячейке GaS (модель $\mathrm{Ga}_{23} \mathrm{~S}_{24}$ )}

Моделирована геометрия структуры атомов в суперьячейке $\mathrm{GaS}$ с вакансией $\mathrm{Ga}$ (модель $\mathrm{Ga}_{23} \mathrm{~S}_{24}$ ) (гексагональная, $a=3.585 \AA, c=15.500 \AA$, пр. гр. $P 6_{3} / m m c$, (194)). Установлено, что длины связи между двумя соседними атомами $\mathrm{Ga}-\mathrm{Ga}, \mathrm{Ga}-\mathrm{S}$ заметно отличаются друг от друга. Длины связи, например, для заданных атомов $\mathrm{Ga}_{10}-\mathrm{Ga}_{21}, \mathrm{Ga}_{11}-\mathrm{S}_{22}$, составляют $d_{\mathrm{Ga}_{10}-\mathrm{Ga}_{21}}=2.47 \AA$, и $d_{\mathrm{Ga}_{11}-\mathrm{S}_{22}}=2.37 \AA$ соответственно.

\section{5. Энергия (энтальпия) образования GaS}

Стабильность дефекта в кристаллической решетке вещества определяется двумя факторами - энергетическим (как результат стремления системы к минимуму свободной энергии) и геометрическим (как выполнение определенных соотношений между эффективными размерами дефекта и соответствующего для него места в кристаллической решетке). Изменение энтальпии образования одного моля данного химического соединения из входящих в него химических элементов в их стандартных состояниях при температуре $T \mathrm{~K}$ и стандартном давлении $1 \mathrm{~atm}(101.3 \mathrm{kPa})$ выражается как

$$
\Delta_{f} H_{T}^{\circ}=\Delta_{f} H_{0}^{\circ}+\int_{0}^{T} \Delta C_{p} d T
$$

где $\Delta_{f} H_{T}^{\circ}-$ стандартная энтальпия образования вещества при $0 \mathrm{~K}, \Delta C_{p}-$ мольная теплоемкость вещества при постоянном давлении ( $p=$ const). (Стандартным состоянием вещества принято считать такое состояние вещества, при котором оно находится в наиболее устойчивой для него форме при стандартном давлении.) С учетом этого стандартную энтальпию образования $\mathrm{GaS}$ при температуре $T$ вычисляли уравнением

$$
\begin{aligned}
& \Delta_{f} H_{T}^{\circ}(\mathrm{GaS})=H_{T}^{\circ}(\mathrm{GaS})-H_{T}^{\circ}(\mathrm{Ga})-H_{T}^{\circ}(\mathrm{S}) \\
& \quad+\int_{0}^{T}\left[C_{p}(\mathrm{GaS})-C_{p}(\mathrm{Ga})-C_{p}(\mathrm{~S})\right] d T \\
& \approx \Delta_{f} H_{0}^{\circ}(\mathrm{GaS})=\Delta_{f} U_{0}^{\circ}(\mathrm{GaS}) \\
& =E_{\mathrm{tot}}^{\circ}(\mathrm{GaS})-\left[E_{\mathrm{tot}}^{\circ}(\mathrm{Ga})+E_{\mathrm{tot}}^{\circ}(\mathrm{S})\right]
\end{aligned}
$$

где $\Delta_{f} H_{T}^{\circ}-$ стандартная энтальпия образования $\mathrm{GaS}$, $\Delta_{f} U_{0}^{\circ}$ - изменение внутренней энергии, $E_{\text {tot }}^{\circ}-$ полная стандартная энергия соединения на формульную единицу GaS. Здесь энтальпии образования простых веществ $\left(H_{T}^{\circ}(\mathrm{Ga})\right.$ и $\left.H_{T}^{\circ}(S)\right)$ равны нулю. С учетом справочных данных $[4,34,35]$ для $\mathrm{GaS}$ получено уравнение $C_{p}(\mathrm{GaS})=41.4+15.7 \cdot 10^{-3} \cdot T \mathrm{~J} /($ моль $\cdot \mathrm{K})$. Мольная теплоемкость оцененная правилом Неймана-Коппа,

\begin{tabular}{|c|c|c|}
\hline \multirow[t]{2}{*}{ Фаза } & \multicolumn{2}{|r|}{$\begin{array}{c}\text { Энтальпии образования, } \\
-\Delta_{f} H_{298}^{\circ} \mathrm{GaS}, \mathrm{kJ} / \mathrm{mol}\end{array}$} \\
\hline & Расчет & Эксперимент \\
\hline \multirow[t]{3}{*}{$\operatorname{GaS}\left(P 6_{3} / m m c\right)$} & \multirow[t]{3}{*}{188.4} & $194.1 \pm 14.6$ [36] (измерение ЭДС) \\
\hline & & $209.2 \pm 20.9$ [35] (калориметрия) \\
\hline & & 196.2 \\
\hline
\end{tabular}
не сильно отличается от суммы атомарных теплоемкостей элементов $C_{p}(\mathrm{Ga})=26.1$ и $C_{p}(\mathrm{~S})=22.6 \mathrm{~J} /(\mathrm{mol} \cdot \mathrm{K})$
Таблица 2. Теоретические и экспериментальные энтальпии образования фазы $\mathrm{GaS}$ в гексагональной структуре

при $298 \mathrm{~K}$ [35]. С учетом этого вклад в уравнение (2) при расчетах энергии образования $\mathrm{GaS}$ принимали равным нулю. В этом случае, энтальпию образования $\mathrm{GaS}$ можно выразить как изменение внутренней энергии $\Delta_{f} U_{0}^{\circ}(\mathrm{GaS})$.

Вычисленные по формуле (4) и экспериментально полученные значения энтальпии образования $\mathrm{GaS}$ приведены в табл. 2. Теоретическая энтальпия образования $\Delta_{f} H_{T}^{\circ}(\mathrm{GaS})=-1.95 \mathrm{eV}$ получена для гексагональной фазы. Это вычисленное значение $\left(\Delta_{f} H_{T}^{\circ}(\mathrm{GaS})=-188.4 \mathrm{~kJ} / \mathrm{mol}\right) \quad$ соответствует справочным экспериментальным данным $\left(\Delta_{f} H_{298}^{\circ}=\right.$ $=-196.2 \mathrm{~kJ} / \mathrm{mol} \quad[3] ; \quad-209.2 \pm 20.9 \mathrm{~kJ} / \mathrm{mol} \quad[35] ;$ $-194.1 \pm 14.6 \mathrm{~kJ} / \mathrm{mol}$ (измерение ЭДС) [36]; $-187.4 \mathrm{~kJ} / \mathrm{mol}$ (калориметрия) [36]), согласно которым гексагональная фаза устойчива при комнатных и низких температурах.

\section{6. Геометрия вокруг вакансий $\mathrm{Ga}$ и $\mathrm{S}$ в суперъячейке GaS}

Рассмотрим результаты оптимизации расположения ближайших соседей атомов вокруг вакансии суперъячейки $\mathrm{GaS}$, состоящей из 48 атомов. Сравнение расстояний между вакансией и атомами соседа до и после оптимизации геометрии показало, что происходят смещения соседних атомов, сопровождаемые вакансиями $\mathrm{Ga}$ и $\mathrm{S}$ (табл. 3). Анализ расстояний между вакансиями $\mathrm{Ga}$ и $\mathrm{S}$ и атомами соседа до и после оптимизации геометрии позволяет сделать следующий вывод.

1. В случае образования вакансии атома Ga в суперьячейке $\mathrm{GaS}$ связи между атомом $\mathrm{Ga}$ и окружающими его атомами $\mathrm{S}$ разрываются. В результате атомы ближайших соседей $\mathrm{S}$ мигрируют в направлении от вакансии $\mathrm{Ga}$. A соседние атомы $\mathrm{Ga}$ движутся в направлении, которое приближается к положению вакансии Ga. Если считать, что связь Ga-S является ковалентной, то когда позиция $\mathrm{Ga}$ будет вакантной, она будет влиять на электронную структуру координированных атомов $\mathrm{S}$.

Из анализа плотности состояний суперъячейки $\mathrm{GaS}$ с вакансиями Ga следует, что основным компонентом верха валентной зоны является орбиталь $\mathrm{S} 3 p$, поэтому 
Таблица 3. Расстояние между вакансией и атомами соседа до и после оптимизации геометрии и смещения атомов соседа с вакансиями $\mathrm{Ga}$ и $\mathrm{S}$

\begin{tabular}{c|c|c|c}
\hline \multirow{2}{*}{$\begin{array}{c}\text { Связь между } \\
\text { вакансией и атомами }\end{array}$} & \multicolumn{3}{|c}{$\begin{array}{c}\text { Расстояние между } \\
\text { вакансией и атомами }\end{array}$} \\
\cline { 2 - 4 } & $l_{\text {befor }}^{a}$ & $l_{\text {after }}^{b}$ & $\Delta l^{c}$ \\
\cline { 2 - 4 } & \multicolumn{3}{|c}{$\AA$} \\
\hline$V_{\mathrm{Ga}}-\mathrm{S}$ & 2.875 & 2.885 & +0.01 \\
$V_{\mathrm{Ga}}-\mathrm{Ga}$ & 2.411 & 2.377 & -0.03
\end{tabular}

Примечание. a) Атомное расстояние до оптимизации геометрии. $b$ ) Атомное расстояние после оптимизации геометрии. $c$ ) Смещение соседних атомов.

акцепторный уровень в запрещенной зоне должен создаваться наверху валентной зоны.

2. В случае вакансии атома $\mathrm{S}$ в суперьячейке $\mathrm{GaS}$ связи между атомом $\mathrm{S}$ и окружающими атомами Ga разрываются. В результате соседние атомы Ga мигрируют в направлении от вакансии. Атомы $\mathrm{S}$ соседа вакансии движутся в направлении, приближающемся к положению вакансии S. Как и в случае (1), когда в кристаллическом узле $\mathrm{S}$ есть вакансия, это влияет на электронную структуру $\mathrm{Ga}$. Поэтому плотность состояний атомов $\mathrm{Ga}$ ближайшего соседа вакансии меняется больше, чем DOS атомы ближайшего соседа Ga в суперъячейке GaS. Анализ DOS суперъячейки $\mathrm{GaS}$ с вакансиями $\mathrm{S}$ указывает на то, что компонентом нижней зоны проводимости является $4 p$-орбиталь $\mathrm{Ga}$, которая создает донорный уровень под нижней частью зоны проводимости.

3. Величина смещения ближайших соседних атомов суперъячейки $\mathrm{GaS}$ с вакансиями $\mathrm{Ga}$ и $\mathrm{S}$, полученная в результате генерации вакансии $\mathrm{Ga}\left(\Delta l\left(V_{\mathrm{Ga}}-\mathrm{S}\right)\right)$, составляет $0.01 \AA$, а величина смещения, определяемая генерацией вакансии $\mathrm{S}\left(\Delta l\left(V_{\mathrm{S}}-\mathrm{Ga}\right)\right)$ составляет $0.03 \AA$. Другими словами влияние вакансии $\mathrm{S}$ на окружающие атомы в $\mathrm{GaS}$ меньше чем влияние вакансии $\mathrm{Ga}$.

\section{7. Энергии образования вакансий Ga и $\mathrm{S}$ B GaS. Химический потенциал}

Структурно-чувствительные свойства (электропроводность, оптические свойства, люминесценция и т.д.), полупроводникового кристалла $\mathrm{GaS}$, сильно зависят от влияния примесей и дефектов [5]. Степень изменения этих свойств определяется концентрацией и типом дефектов. Одним из основных параметров, характеризующих точечный дефект, является энергия образования $E_{f}^{\mathrm{def}}$ дефекта.

Процесс образования вакансий $\mathrm{Ga}$ и $\mathrm{S}$ в суперьячейке $\mathrm{GaS}$ можем отнести к гомогенным открытым термодинамическим системам, в которых масса и состав системы изменяются за счет выведения или добавления вещества, или за счет протекания химической реакции. В открытых термодинамических системах функции состояния системы, а именно внутренняя энергия $U$, энтальпия $H$, энергия Гельмгольца $F$ и энергия Гиббса $G$ являются также функцией количества образующих данную систему индивидуальных компонентов. Удаление некоторого количества вещества из открытой системы или появление его в системе будет влиять на запас внутренной энергии системы.

В изохорно- (или изобарно)-изотермических условиях приращение свободной энергии Гельмгольца $(\Delta F)$ или Гиббса $(\Delta G)$ открытой системы при добавлении к ней одного моля этого компонента равен химическому потенциалу $\mu_{i}$. Химический потенциал определяется одной из следующих частных производных:

$$
\begin{aligned}
\mu_{i} & =\left(\partial U / \partial n_{i}\right)_{S, V, n_{j \neq i}}=\left(\partial H / \partial n_{i}\right)_{S, P, n_{j \neq i}} \\
& =\left(\partial F / \partial n_{i}\right)_{V, T, n_{j \neq i}}=\left(\partial G / \partial n_{i}\right)_{P, T, n_{j \neq i}},
\end{aligned}
$$

где $n_{i}$ - число молей всех компонентов, кроме того компонента, изменение которого рассматривается. Величина $\mu_{i}$ характеризует степень изменения полной энергии, в частности, свободной энергии Гиббса $\Delta G$ системы при $T=$ const, $P=$ const и добавлении к ней бесконечно малого количества компонента $i$ без изменения числа молей других компонентов $j$. Т.е. в этом случае количество остальных веществ в этой ситеме в расчете на один моль остается постоянным. Поэтому химический потенциал $i$-го компонента совпадает с парциальным изобарным потенциалом $\left(\mu_{i}=\overline{G_{i}}\right)$ одного моля вещества. Другими словами принимается, что открытая система настолько велика, что ее состав при добавлении к ней одного моля $i$-го компонента практически не меняется.

С другой стороны химический потенциал $i$-го компонента определяется известным соотношением: $\mu_{i}=\mu_{i}^{0}+R T \ln \left(x_{i}\right)$ или $\mu_{i}=\overline{H_{l}^{0}}-T \overline{S_{l}^{0}}$, где $\mu_{i}^{0}-$ стан- $^{-}$ дартный химический потенциал $i$-го компонента в чистой фазе, $R$ - универсальная газовая постоянная, $\overline{H_{l}^{0}}$ и $\overline{S_{l}^{0}}$ стандартные мольные парциальные энтальпия и энтропия. Отсюда следует, что химический потенциал атома изменяется в зависимости от химического состава. $\mathrm{B}$ частности, в суперьячейке $\mathrm{GaS}$ с вакансией величина $\mu_{i}$ должна зависеть от состава соединения $\mathrm{GaS}$.

Рассмотрим связь энергии образования дефектов $E_{f}^{\mathrm{def}}$ со свободной энергией системы в процессе образования $n$ - числа дефектов в кристалле. В изотермическом обратимом процессе, работа, совершенная системой равна уменьшению $\Delta F(V=$ const $)$ или $\Delta G(P=$ const $)$. С другой стороны $E_{f}^{\mathrm{def}}$ определяется работой по переносу атома из узла кристаллической решетки на поверхность кристалла. В условиях термодинамического равновесия образование точечных дефектов будет повышать энтропию $(S)$ кристалла. При этом из кристалла, содержащего $N$ одинаковых атомов, можно удалить $n$ атомов различными способами. Т.е. в кристалле из $N$ атомов в $N$ позициях кристалла число молекул в 1 моле $N_{A}=6.02 \cdot 10^{23}$ - число Авогадро) можно создать $n$ 
количество дефектов $(n \ll N)$. Тогда согласно формуле Больцмана соответствующее изменение конфигурационной энтропии равно: $\Delta S_{c}=k_{\mathrm{B}} \ln W=k_{\mathrm{B}} \ln \left[\frac{N !}{n !(N-n)}\right]$, где $k_{\mathrm{B}}-$ постоянная Больцмана. Здесь $k_{\mathrm{B}} T$ характеризует энергию „теплового“ кванта, равного $0.026 \mathrm{eV}$ при $298 \mathrm{~K}$. В равновесном случае образование $n$-го числа дефектов при температуре $T$ будет изменять $\Delta F$ или $\Delta G$ кристалла в соответствии с уравнением ГиббсаГельмгольца для процесса: $\Delta F=n \Delta_{f} U^{\mathrm{def}}-T \Delta S$ или $\Delta G=n \Delta_{f} H^{\text {def }}-T \Delta S, \quad$ где $\Delta S=S_{c}+n S_{v}^{\text {def }}$ (изменение колебательной энтропии при образовании одного дефекта). Здесь $\Delta_{f} U^{\mathrm{def}} \approx \Delta_{f} H^{\mathrm{def}}=E_{f}^{\mathrm{def}}-$ энергия (энтальпия или теплота) образования одного дефекта. Отсюда следует, что $\Delta_{f} H^{\text {def }}$ при малых $n$ мало. Заметный вклад $T \Delta S$ определяется возрастанием конфигурационной энтропии $\Delta S_{c}=k_{\mathrm{B}} \ln W$.

Минимизация $\Delta F$ или $\Delta G$ с учетом $\Delta S$ и факториалов чисел $\ln (n !) \approx n \ln (n)$ позволяет оценить равновесное число точечных дефектов $n=N \exp \left(-\Delta_{f} H^{\mathrm{def}} / k_{\mathrm{B}} T\right)$, a также равновесную концентрацию дефектов $C^{\text {def }}=\exp \left(S_{v} / k_{\mathrm{B}}\right) \cdot \exp \left(-\Delta_{f} H^{\mathrm{def}} / k_{\mathrm{B}} T\right) . \quad$ Энтропийные вклады в энергию образования дефекта не учитывали в расчетах. Во-первых, использовали суперьячейки с моновакансиями, где общая концентрация дефектов значительно ниже $10^{-4}$, а конфигурационная энтропия на дефект больше $10 k_{\mathrm{B}}$ и $S_{c}^{\text {def }}$ поэтому может быть доминирующим вкладом энтропии при высоких температурах. Во-вторых, учет незначительных вкладов колебательной и магнитной энтропии увеличивает вычислительные затраты на несколько порядков по сравнению со статическим $(T=0 \mathrm{~K})$ расчетом дефекта. Энергия образования дефекта в зарядовом состоянии $q$ равна [37]:

$$
E_{f}^{\mathrm{q} . \mathrm{def}}=E_{\mathrm{tot}}^{\mathrm{def}}-E_{\mathrm{tot}}^{\mathrm{bulk}}+\sum_{i} n_{i} \mu_{i}+q\left(E_{\mathrm{F}}+E_{\mathrm{VBM}}\right)+Q_{c},
$$

где $E_{\mathrm{tot}}^{\mathrm{def}}-$ полная энергия суперъячейки, содержащей дефект, $E_{\mathrm{tot}}^{\mathrm{bulk}}$ - полная энергия объемной суперьячейки, $\mu_{i}$ - химический потенциал частиц $i$, добавленных $\left(n_{i}>0\right)$ или удаленных $\left(n_{i}<0\right)$ из ячейки для создания дефекта, $E_{\mathrm{F}}-$ энергия Ферми, $E_{\mathrm{VBM}}-$ максимум валентной зоны в объеме, так что $E_{\mathrm{F}} \geq 0, Q_{c}-$ электростатические поправки, которые учитывают дальнодействующие электростатические взаимодействия заряженных дефектов соседних суперьячеек. Величина $\mu_{i}$ влияет на энергию образования дефекта, но не на энергетические уровни перехода частиц. Уровень ионизации дефекта, характеризующий степень термодинамического перехода между зарядовыми состояниями $q_{1}$ и $q_{2}$ определяется как [37]:

$$
\epsilon\left(q_{1} / q_{2}\right)=\frac{\left.E_{\mathrm{F}}^{q_{1}}\right|_{E_{\mathrm{F}=0}}-\left.E_{\mathrm{F}}^{q_{2}}\right|_{E_{\mathrm{F}}=0}}{q_{1}-q_{2},}
$$

где $\left.E_{\mathrm{F}}^{q}\right|_{\mathrm{F}}=0-$ энергия образования дефекта в зарядовом состоянии $q$, оцененная при $E_{\mathrm{F}}=0$. Примем, что $q=0$ и условие электронейтральности в $\mathrm{GaS}$ обеспечивается образованием нейтральных вакансий собственных атомов $\mathrm{Ga}$ и $\mathrm{S}$. Энергию образования $\Delta_{f} H^{\mathrm{V}}$ или $E_{f}^{\mathrm{V}}$ нейтральных вакансий галлия $\left(\mathrm{V}_{\mathrm{Ga}}^{\mathrm{X}}\right)$ и серы $\left(\mathrm{V}_{\mathrm{S}}^{\mathrm{X}}\right)$ в соответствующих подрешетках суперъячеек $\mathrm{GaS}$ вычисляли выражениями вида [30]:

$$
\begin{gathered}
E_{f}^{\mathrm{V}_{\mathrm{Ca}}^{\mathrm{X}}}=E_{\mathrm{tot}}^{\mathrm{V}_{\mathrm{Ga}}^{\mathrm{X}}+\text { bulk }}\left(\mathrm{Ga}_{n-1} \mathrm{~S}_{n}\right)-E_{\mathrm{tot}}^{\mathrm{bulk}}\left(\mathrm{Ga}_{n} \mathrm{~S}_{n}\right)+\mu_{\mathrm{Ga}}, \\
E_{f}^{\mathrm{V}_{\mathrm{S}}^{\mathrm{X}}}=E_{\mathrm{tot}}^{\mathrm{V}_{\mathrm{S}}^{\mathrm{X}}+\text { bulk }}\left(\mathrm{Ga}_{n} \mathrm{~S}_{n-1}\right)-E_{\mathrm{tot}}^{\text {bulk }}\left(\mathrm{Ga}_{n} \mathrm{~S}_{n}\right)+\mu_{\mathrm{S}},
\end{gathered}
$$

где $E_{\mathrm{tot}}^{\mathrm{V}_{\mathrm{a}}^{\mathrm{X}}+\text { bulk }}$ - полная энергия суперъячейки объемного вещества (bulk) с нейтральной вакансией галлия или серы $\left(\mathrm{V}_{\mathrm{Ga}, \mathrm{S}}^{\mathrm{X}}\right), E_{\mathrm{tot}}^{\text {fulk }}-$ полная энергия суперъячейки объемного вещества (bulk) без нейтральной вакансии, $n$ - количество атомов в суперъячейке $\mathrm{GaS}, \mu_{i}-$ химический потенциал $i$-го компонента.

Рассмотрим зависимость $\mu_{i}$ от состава в GaS. Примем, что как и другие халькогенидные соединения типа $\mathrm{A}^{\mathrm{III}} \mathrm{B}^{\mathrm{VI}}[3], \mathrm{GaS}$ имеет малое отклонение от стехиометрии, т.е. имеет нестехиометрический состав. Тогда процесс образования твердого растворов $\mathrm{Ga}_{x} \mathrm{~S}_{x-1}$ или $\mathrm{Ga}_{x-1} \mathrm{~S}_{x}$ будет сопровождаться изменением свободной энергии Гиббса $(\Delta G)$. Для энергии смешения $\Delta G$ одного моля раствора можно написать: $\Delta G=x_{\mathrm{Ga}} \mu_{\mathrm{Ga}}+x_{\mathrm{S}} \mu_{\mathrm{S}}$, где $x_{i}$ - мольная доля компонента $i$ в растворе.

Для стехиометрического состава $\mathrm{GaS}$ должно соблюдаться равенство: $\mu_{\mathrm{Ga}}+\mu_{\mathrm{S}}=\mu_{\mathrm{GaS}(\mathrm{bulk})}$, где $\mu_{\mathrm{GaS}(\mathrm{bulk})}$ химический потенциал для объемного соединения $\mathrm{GaS}$. Если принять, что состав $\mathrm{GaS}$ незначительно отклоняется от стехиометрии, тогда необходимо согласовать значения химических потенциалов атомов в области гомогенности GaS. C этой целью фигуративные точки (составы) для расчета $\mu_{i}$ атомов выбрали из $T-x$ фазовой диаграммы системы Ga-S [3,36].

Уточненная нами $T-x$ диаграмма Ga-S приведена на рис. 9. На линии солидуса $T-x$ диаграммы вблизи соединения $\mathrm{GaS}$, кроме стехиометрического состава, выбирали по одному составу $\mathrm{Ga}_{0.501} \mathrm{~S}_{0.499}$ слева и $\mathrm{Ga}_{0.499} \mathrm{~S}_{0.501}$ справа от стехиометрического состава $\mathrm{GaS}$, для которых рассчитывали $\mu_{i}$. Выбранные составы на $T-x$-диаграмме $\mathrm{Ga}-\mathrm{S}$, характеризуют равновесие $\mathrm{GaS}$ в гетерогенных областях $\mathrm{Ga}_{2} \mathrm{~S}-\mathrm{GaS}$ и $\mathrm{GaS}-\mathrm{Ga}_{2} \mathrm{~S}_{3}$ с соединениями $\mathrm{Ga}_{2} \mathrm{~S}$ и $\mathrm{Ga}_{2} \mathrm{~S}_{3}$, сответственно.

Исходя из указанного и с учетом $T-x$ диаграммы, $\mathrm{Ga}-\mathrm{S}$ для стехиометрического состава $\mathrm{GaS}$, химический потенциал атомов $\mathrm{Ga}$ и $\mathrm{S}$ можно задать следующим образом:

$$
\begin{gathered}
\mu_{\mathrm{Ga}}=\left[\mu_{\mathrm{Ga}_{2} \mathrm{~S}(\text { bulk })}-\mu_{\mathrm{GaS}(\text { bulk })}\right], \\
\mu_{\mathrm{Ga}}=\left[3 \mu_{\mathrm{GaS}(\text { bulk })}-\mu_{\mathrm{Ga}_{2} \mathrm{~S}_{3}(\text { bulk })}\right], \\
\mu_{\mathrm{S}}=\mu_{\mathrm{S}(\text { bulk })} .
\end{gathered}
$$

Химические потенциалы объемных веществ ( $\mathrm{GaS}$, $\mathrm{Ga}_{2} \mathrm{~S}$ и $\left.\mathrm{Ga}_{2} \mathrm{~S}_{3}\right)$ в этих уравнениях рассчитаны как полные энергии на формульную единицу путем отдельных расчетов: $\mu_{\mathrm{GaS}}=4.9 \mathrm{eV}, \mu_{\mathrm{Ga}_{2} \mathrm{~S}}=7.3 \mathrm{eV}, \mu_{\mathrm{Ga}_{2} \mathrm{~S}_{3}}=12.9 \mathrm{eV}$. 


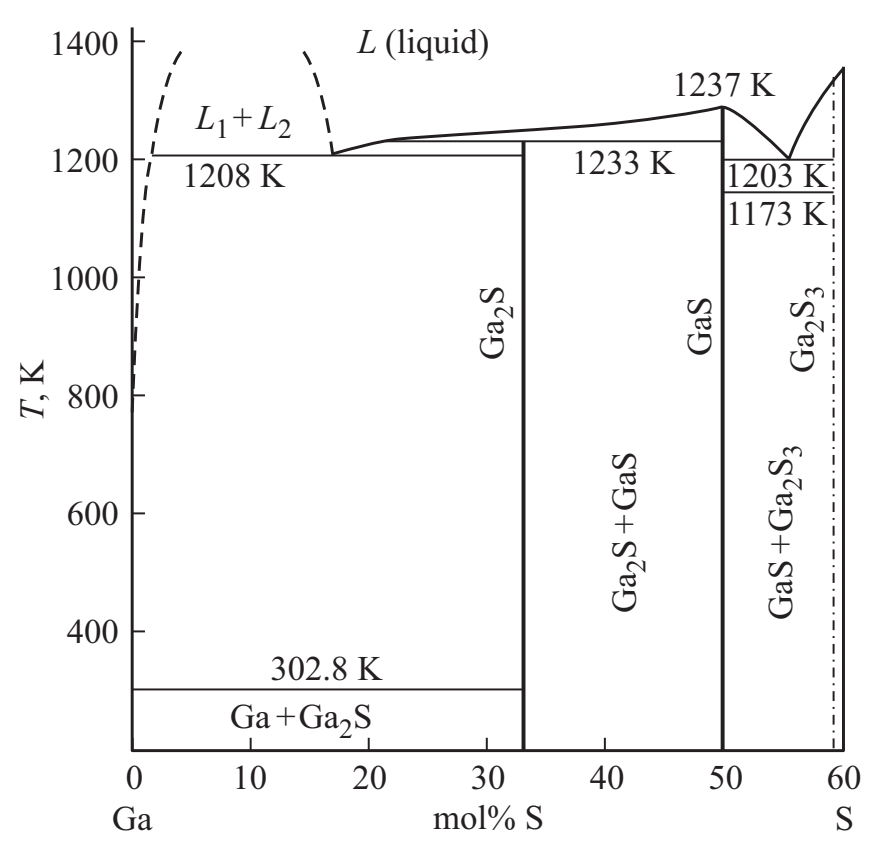

Рис. 9. Фазовая диаграмма состояния системы Ga-S в интервале концентраций 0-60 ат.\% серы.

DFT-рассчитанные нами значения $\mu_{\mathrm{Ga}}$ и $\mu_{\mathrm{S}}$ отличаются на $\sim 0.02 \mathrm{eV}$ от справочных значений $\mu_{\mathrm{Ga}}=2.42$ и $\mu_{\mathrm{S}}=2.45 \mathrm{eV}[38]$ в газовом состоянии элементов. Изменения $\mu_{i}$ составов $\mathrm{Ga}_{0.501} \mathrm{~S}_{0.499}$ и $\mathrm{Ga}_{0.499} \mathrm{~S}_{0.501}$ в выбранной области гомогенности $\mathrm{GaS}$ находятся также в пределах погрешности $\sim 0.02 \mathrm{eV}$. С учетом $\mu_{i}$ веществ $\left(\mathrm{GaS}, \mathrm{Ga}_{2} \mathrm{~S}\right.$ и $\left.\mathrm{Ga}_{2} \mathrm{~S}_{3}\right)$ рассчитанные энергии образования вакансий $\mathrm{Ga}$ и $\mathrm{S}$ в суперъячейках $\mathrm{GaS}$ составляли: $E_{f}^{\mathrm{V}_{\mathrm{G} a}^{x}}=0.57$ и $E_{f}^{\mathrm{V}_{\mathrm{S}}^{x}}=0.62 \mathrm{eV}$.

$\mathrm{B} \mathrm{GaS}$ энергия образования Ga-вакансии меньше, чем энергия образования вакансий S. T.е. поскольку энергии образования вакансий $\mathrm{S}$ в соединении $\mathrm{GaS}$ больше $\left(E_{f}^{\mathrm{V}_{\mathrm{S}}^{x}}>E_{f}^{\mathrm{V}_{\mathrm{G} a}^{x}}\right)$, то возникновение вакансии $\mathrm{S}$ затруднено. Это согласуется с тем, что когда поликристаллы $\mathrm{GaS}$ синтезируются непосредственно сплавлением стехиометрического количества $\mathrm{Ga}$ и $\mathrm{S}$ в вакууме, легко создаются Ga-вакансии. C другой стороны, в условиях недостатка серы $\left(\mathrm{Ga}_{n} \mathrm{~S}_{n-1}\right)$ энергия образования вакансии $\mathrm{S}$ составляет $\sim 0.62 \mathrm{eV}$. Следовательно, для $\mathrm{Ga}_{n} \mathrm{~S}_{n-1}$ генерировать вакансию $\mathrm{S}$ несложно. Вакансию $\mathrm{S}$ в $\mathrm{GaS}$ можно устранить термообработкой в окислительной атмосфере при температурах $\leq 1100 \mathrm{~K}$. Синтезированные нами поликристаллы $\mathrm{GaS}$ высокой плотности после их отжига в окислительной атмосфере, т.е. в незначительном избытке серы, имели стехиометрический состав. Энергия образования вакансии $\mathrm{Ga}$ является максимальной как в составах богатых серой, так и в стехиометрическом составе $\mathrm{GaS}$.

B GaS с точечным дефектом (вакансия) рассмотрим зависимость энергии образования дефектов $E_{f}^{\mathrm{V}}$ от химического потенциала $\mu$. Величина $E_{f}^{\mathrm{V}}$ и следовательно, равновесная концентрация дефектов сильно зависят от атомных химических потенциалов $\mathrm{S}$ и $\mathrm{Ga}$, а также от химического потенциала электронов в термодинамически разрешенном диапазоне. В условиях равновесии и электронейтральности для расчета $E_{f}^{\mathrm{V}}$ в объеме $\mathrm{GaS}$ на химические потенциалы применим следующие ограничения. Если система содержит избыток серы, то этот избыток $\mathrm{S}$ может осаждаться в объеме $\mathrm{GaS}$. Следовательно, химический потенциал избытка $\mathrm{S}$ не может превышать химический потенциал серы в объеме, т.е. $\mu_{\mathrm{S} \text { (excess) }} \leq \mu_{\mathrm{S} \text { (bulk) }}$. Точно так же химический потенциал избытка $\mathrm{Ga}$ не может превышать химический потенциал галлия в объеме $\mu_{\mathrm{Ga} \text { (bulk) }}$, т. е. $\mu_{\mathrm{Ga} \text { (excess) }} \leq \mu_{\mathrm{S} \text { (bulk) }}$. Кроме того, требуется, чтобы сумма химических потенциалов $\mathrm{S}$ и $\mathrm{Ga}$, равнялось химическому потенциалу (на пару) объемного GaS:

$$
\begin{aligned}
\mu_{\mathrm{S}}+\mu_{\mathrm{Ga}} & =\mu_{\mathrm{GaS}(\text { bulk })} \\
& =\mu_{\mathrm{Ga}(\text { bulk })}+\mu_{\mathrm{S}(\text { bulk })}-\Delta_{f} H(\mathrm{GaS}(\text { bulk })),
\end{aligned}
$$

где $\Delta_{f} H-$ энтальпия образования GaS.

Другими словами используя указанные выше ограничения для индивидуального $\mu_{i}$, который задается объемными элементами $\mathrm{S}$ и $\mathrm{Ga}$, обнаруживается, что поверхность $\mathrm{GaS}$ может находиться в равновесии с окружающей средой системы только в том случае, если химические потенциалы находятся в пределах как верхнего, так и нижнего пределов.

$$
\begin{gathered}
\mu_{\mathrm{S} \text { (bulk) }}-\Delta_{f} H \leq \mu_{\mathrm{S}} \leq \mu_{\mathrm{S}(\text { bulk })}, \\
\mu_{\mathrm{Ga} \text { (bulk) }}-\Delta_{f} H \leq \mu_{\mathrm{Ga}} \leq \mu_{\mathrm{Ga}(\text { bulk })} .
\end{gathered}
$$

Таким образом, каждый $\mu_{i}$ ограничен диапазоном на $\sim 2 \mathrm{eV} \quad\left(-\Delta_{f} H(\mathrm{GaS})=2.17 \mathrm{eV} \quad[35] ; 1.97 \mathrm{eV} \quad[36]\right.$, $2.03 \mathrm{eV}$ [3] ниже его соответствующего объемного значения.

Эти условия ограничивают разность химических потенциалов $\mu_{\mathrm{Ga}}-\mu_{\mathrm{s}}$, диапазоном, определяемым теплотой образования $\Delta_{f} H$ объемного $\mathrm{GaS}$ из элементарных $\mathrm{S}$ и $\mathrm{Ga}$. C учетом указанных условий, энергию образования дефекта $E_{f}^{\mathrm{V}}$ в суперъячейке $\mathrm{GaS}$ с одним дефектом (вакансия) можно записать как

$$
E_{f}^{\mathrm{V}}=E_{D}-n_{e} \mu_{e}-n_{\mathrm{Ga}} \mu_{\mathrm{Ga}}-n_{\mathrm{S}} \mu_{\mathrm{S}},
$$

где $E_{D}$ - энергия суперъячейки, содержащей $n_{\mathrm{Ga}}$ атомов $\mathrm{Ga}, n_{\mathrm{S}}$ атомов $\mathrm{S}$ и один дефект (вакансия). Здесь $n_{e}$ - количество электронов, перенесенных из электронного резервуара системы (т. е. система состоит из сколь угодно большого числа частиц) с химическим потенциалом $\mu_{e}$ в дефект для данного зарядового состояния.

Определив

$$
\Delta \mu=\left(\mu_{\mathrm{Ga}}-\mu_{\mathrm{S}}\right)-\left(\mu_{\mathrm{Ga}(\mathrm{bulk})}-\mu_{\mathrm{S}(\mathrm{bulk})}\right)
$$

величину $E_{f}^{\mathrm{v}}$ перепишем как

$$
E_{f}^{\mathrm{V}}=E_{D}-n_{e} \mu_{e}-\frac{1}{2}\left(n_{\mathrm{Ga}}-n_{\mathrm{S}}\right) \Delta \mu,
$$

где $E_{D}$ не зависит от $\Delta \mu$ и $\mu_{e}$. 
Для идеального кристалла вещества величина $E_{D}^{\mathrm{id}}=0$, и энергия образования $\Delta_{f} H$ вещества обращается в нуль. Для объемного $\mathrm{GaS}$, если применить ограничения на химические потенциалы $0 \leq \mu_{e} \leq E_{g}$ и $-\Delta_{f} H \leq \Delta \mu \leq \Delta_{f} H$, где $E_{g}-$ запрещенная зона, тогда теплота образования $\mathrm{GaS}$ будет равна $\Delta_{f} H=-\left(\mu_{\mathrm{GaS}}-\mu_{\mathrm{Ga}(\text { bulk })}-\mu_{\mathrm{S}(\mathrm{bulk})}\right)$. В таких расчетах можно пренебрегать температурной зависимостью $E_{D}$, $E_{g}$ и $\Delta_{f} H$. Однако, температурная зависимость является заметной для получения точных концентраций дефектов при высоких температурах.

\section{4. Заключение}

Установлено, что полученные монокристаллы $\mathrm{GaS}$ (гексагональной структуры пр. гр. $P 6_{3} / m m c: a=3.583 \AA$, $c=15.475 \AA$ ) обладают высоким удельным сопротивлением и запрещенной зоной $\Delta E_{g}=2.5 \mathrm{eV}$ при $300 \mathrm{~K}$. Частотные зависимости проводимости на переменном токе поперек слоев монокристалла $\mathrm{GaS}$ до и после электронного облучения различными дозами указывают на то, что в результате облучения проводимость $\mathrm{GaS}$ уменьшается. Уменьшение электропроводности облученного $\mathrm{GaS}$ можно связать с компенсацией первоначально существовавших электрически активных примесных центров глубокими энергетическими уровнями радиационных дефектов. Таким образом, облучение монокристалла $\mathrm{GaS}$ вызывает возникновение радиационных дефектов и стимулирует отжиг и миграцию имеющихся в кристалле дефектов.

DFT-расчеты зонной и электронной структуры в дефектных суперъячейках $\mathrm{GaS}$ с 36 и 48 атомами позволили определить вид электронной плотности и согласовать значения $\Delta E_{g}$ между расчетом и экспериментом. Установлено, что рассчетная энтальпия образования фазы $\mathrm{GaS}\left(-\Delta_{f} H_{T}^{\circ}(\mathrm{GaSd})=1.95 \mathrm{eV}\right.$ (или $\left.-188.4 \mathrm{~kJ} / \mathrm{mol}\right) \quad$ с использованием метода DFT соответствует экспериментальному результату, согласно которому гексагональная фаза ((пр.гр.)P6 $3 / m m c)$ устойчива при низких температурах. DFT-расчет также позволил оценить степень влияния разупорядоченных нейтральных вакансий атомов на свойства GaS. Энергии образования нейтральных вакансий в $\mathrm{GaS}$ с учетом химических потенциалов $\mathrm{Ga}$ и $\mathrm{S}$ согласуются с литературными данными.

Энергия образования вакансии $\mathrm{Ga}\left(E_{f}^{\mathrm{V}_{\mathrm{Ga}}^{x}}=0.57 \mathrm{eV}\right)$ ниже, чем у вакансий $\mathrm{S}\left(E_{f}^{\mathrm{V}_{\mathrm{S}}^{x}}=0.62 \mathrm{eV}\right)$ в окислительной атмосфере. Энергия образования вакансии $\mathrm{S}$ ниже, чем у вакансий $\mathrm{Ga}$ в восстановительной атмосфере. Отсюда следует, что физические свойства (электрические, оптических и т.д.) существенно зависят от наличия дефектов в слоистом кристалле GaS. Это происходит также за счет разупорядочения атомов $\mathrm{Ga}$ и $\mathrm{S}$ по кристаллографически неэквивалентным позициям гексагональной структуры GaS. Полученные результаты могут быть использованы для получения 2D-материалов на основе $\mathrm{GaS}$ с воспроизводимыми свойствами.

\section{Благодарности}

Работа выполнена при частичной поддержке Фонда развития науки при Президенте Азербайджанской Республики (EIF) (грант № E IF-BGM-3-BRFTF-2+/201715/05/1-M-13) и Российского фонда фундаментальных исследований (РФФИ) (проект № Az_a2018).

\section{Конфликт интересов}

Авторы заявляют, что у них нет конфликта интересов.

\section{Список литературы}

[1] S.M. Asadov S.N. Mustafaeva, V.F. Lukichev. Russ. Microelectron. 48, 422 (2019). https://doi.org/10.1134/S1063739719660016

[2] S.N. Mustafaeva, M.M. Asadov, A.A. Ismailov. Phys. Solid State 50, 2040 (2008). https://doi.org/10.1134/S1063783408110073

[3] Н.Х. Абрикосов, В.Ф. Банкина, Л.В. Порецкая, Е.В. Скуднова, С.Н. Чижевская. Полупроводниковые халькогениды и сплавы на их основе. Наука, М. (1975). 220 с.

[4] Semiconductors Data Handbook / Ed. O. Madelung. Springer, Berlin (2004). https://doi.org/10.1007/978-3-642-18865-7

[5] S.N. Mustafaeva, M.M. Asadov. Solid State Commun. 45, 491 (1983). https://doi.org/10.1016/0038-1098(83)90159-X

[6] D. Wickramaratne, F. Zahid, R.K. Lake. J. Appl. Phys. 118, 7, 075101 (2015). doi:10.1063/1.4928559

[7] A. Kuhn, A. Bourdon, J. Rigoult, A. Rimsky. Phys. Rev. B 25, 4081 (1982). https://doi.org/10.1103/PhysRevB.25.4081

[8] Z.A. Dzhakhangirli. Phys. Solid State 54, 1092 (2012). https://doi.org/10.1134/S106378341205008

[9] V.V. Karpov, A.V. Bandura, R.A. Evarestov. Phys. Solid Solid State 62, 1017 (2020). https://doi.org/10.1134/s1063783420060116

[10] S.N. Mustafaeva, M.M. Asadov. Mater. Chem. Phys. 15, 185 (1986). https://doi.org/10.1016/0254-0584(86)90123-9

[11] G. Micocci, A. Serra, A. Tepore. J. Appl. Phys. 82, 2365 (1997). https://doi.org/10.1063/1.366046

[12] S. Shigetomi, T. Ikar. J. Appl. Phys. 95, 6480 (2004). https://doi.org/10.1063/1.1715143

[13] Y.-K. Hsu, C.-S. Chang, W.-C. Huang. J. Appl. Phys. 96, 1563 (2004). https://doi.org/10.1063/1.1760238

[14] M.M. Asadov, S.N. Mustafaeva, S.S. Guseinov, V.F. Lukichev. Phys. Solid State 62, 2224 (2020). https://doi.org/10.1134/S1063783420110037

[15] Inorganic Nanosheets and Nanosheet-Based Materials. Fundamentals and Applications of Two-Dimensional Sys tems / Eds T. Nakato, J. Kawamata, S. Takagi. Springer, Jpn KK (2017). 542 p. ISBN 978-4-431-56494-2

[16] Y. Ni, H. Wu, C. Huang, M. Mao, Z. Wang, X. Cheng. J. Cryst. Growth. 381, 10 (2013). https://doi.org/10.1016/j.jcrysgro.2013.06.030

[17] T. Wang, J. Li, Q. Zhao, Z. Yin, Y. Zhang, B. Chen, Y. Xie, W. Jie. Materials 11, 186 (2018). https://doi.org/10.3390/ma11020186

[18] S.M. Asadov, S.N. Mustafaeva, A.N. Mammadov. J. Therm. Anal. Calorim. 133, 1135 (2018). https://doi.org/10.1007/s10973-018-6967-7

[19] H.L. Zhuang, R.G. Hennig. Chem. Mater. 25, 3232 (2013). https://doi.org/ 10.1021/cm401661x 
[20] Y. Ma, Y. Dai, M. Guo, L. Yu, B. Huang. Phys. Chem. Chem. Phys. 15, 7098 (2013). https://doi.org/10.1039/C3CP50233C

[21] V. Zólyomi, N.D. Drummond, V.I. Fal'ko. Phys. Rev. B 87, 195403 (2013). https://doi.org/10.1103/PhysRevB.87.195403

[22] C.S. Jung, F. Shojaei, K. Park, J.Y. Oh, H.S. Im, D.M. Jang, J. Park, H.S. Kang. ACS Nano 9, 9585 (2015). https://doi.org/10.1021/acsnano.5b04876

[23] E.G. Seebauer, M.C. Kratzer. Charged Semiconductor Defects. Structure, Thermodynamics and Diffusion. SpringerVerlag London (2009). 294 p. ISBN 978-1-84882-058-6.

[24] A. Alkauskas, P. Deák, J. Neugebauer, A. Pasquarello, C.G. Van de Walle. Advanced Calculations for Defects in Materials (Electronic Structure Methods). Wiley-VCH Verlag \& Co. KGaA, Boschstr. Weinheim, Germany (2011). 384 p. ISBN: 978-3-527-41024-8.

[25] R.A. Evarestov. Theoretical Modeling of Inorganic Nanostructures. 2nd ed.Springer. Nature. Switzerland AG. (2020). 857 p. ISBN 978-3-030-42993-5

[26] H. Chen, Y. Li, L. Huang, J. Li. RSC Adv. 5, 50883 (2015). https://doi.org/10.1039/C5RA08329J

[27] J.P. Perdew, W. Yue. Phys. Rev. B 33, 8800 (1986). https://doi.org/10.1103/physrevb.33.8800

[28] J.P. Perdew, J.A. Chevary, S.H. Vosko, K.A. Jackson, M.R. Pederson, D.J. Singh, C. Fiolhais. Phys. Rev. B. 46, 6671 (1992). https://doi.org/10.1103/physrevb.46.6671

[29] J.P. Perdew, K. Burke, M. Ernzerhof. Phys. Rev. Lett. 77, 3865 (1996). https://doi.org/10.1103/physrevlett.77.3865

[30] M.M. Asadov, S.N. Mustafaeva, S.S. Guseinova, V.F. Lukichev, D.B. Tagiev. Phys. Solid State 63, 797 (2021). https://doi.org/10.1134/S1063783421050036

[31] H.J. Monkhorst. Phys. Rev. B 13, 5188 (1976). http://dx.doi.org/10.1103/PhysRevB.13.5188

[32] W.H. Press, S.A. Teukolsky, W.T. Vetterling, B.P. Flannery. Numerical Recipes. 3nd ed. Cambridge University Press, Cambridge (2007). 490 p. ISBN-13 978-0-511-33555-6

[33] P. Perdew, W. Yang, K. Burke, Z. Yang, E.K.U. Gross, M. Schefflerg, G.E. Scuseria, T.M. Henderson, I.Y. Zhang, A. Ruzsinszky, H. Peng, J. Sun, E. Trushin, A. Görling. Proc. Nat. Acad. Sci. 114, 2801 (2017).

https://doi.org/10.1073/pnas.1621352114

[34] I. Barin, O. Knacke, O. Kubaschewski. Thermochemical properties of inorganic substances. Supplement, Springer-Verlag, Berlin. Heidelberg GmbH (1977). P. 270. ISBN 978-3-662-02295-5

[35] O. Kubashewski, C.B. Alcock, P.J. Spencer. Mater. Thermochem. Pergamon. 6th ed. Press, Oxford, N. Y. (1993). 363 p. ISBN-13: 978-0080418896

[36] А.С. Аббасов Термодинамические свойства некоторых полупроводниковых веществ. Изд-во Элм., Баку (1981). $88 \mathrm{c}$.

[37] C.G. Van de Walle, A. Janotti. Advances in Electronic Structure Methods for Defects and Impurities in Solids / Eds A. Alkauskas, P. Deák, J. Neugebauer, A. Pasquarello, C.G. Van de Walle. Advanced Calculations for Defects in Materials (Electronic Structure Methods). Wiley-VCH Verlag \& Co. KGaA, Weinheim, Germany (2011). P. 1-16. ISBN:978-3-527-41024-8.

[38] G. Job, R. Rüffler. Physikalische Chemie. Vieweg + Teubner Verlag. Springer Fachmedien Wiesbaden GmbH. (2011). ISBN 978-3-8351-0040

Редактор Т.Н. Василевская 